\title{
Self-similarity and Reynolds number invariance in Froude modelling
}

\begin{abstract}
This review aims to improve the reliability of Froude modelling in fluid flows where both the Froude number and Reynolds number are a priori relevant. Two concepts may help to exclude significant Reynolds number scale effects under these conditions: (i) self-similarity and (ii) Reynolds number invariance. Both concepts relate herein to turbulent flows, thereby excluding self-similarity observed in laminar flows and in non-fluid phenomena. These two concepts are illustrated with a wide range of examples: (i) irrotational vortices, wakes, jets and plumes, shear-driven entrainment, high-velocity open channel flows, sediment transport and homogeneous isotropic turbulence and (ii) tidal energy converters, complete mixing in contact tanks and gravity currents. The limitations of self-similarity and Reynolds number invariance are also highlighted. Many fluid phenomena with the limitations under which self-similarity and Reynolds number invariance are observed are summarised in tables, aimed at excluding significant Reynolds number scale effects in physical Froude-based models.
\end{abstract}

Keywords: Froude similarity, Laboratory studies, Reynolds number invariance, Scale effect, Scale invariance, Self-similarity, Similarity (scaling) theory.

\section{Introduction}

\subsection{Context of Froude modelling}

Most physical model studies in hydraulic engineering involving free surface flows are modelled with Froude similarity. Froude similarity is based on the assumption $\mathrm{F}_{M}=\mathrm{F}_{P}$ (subscript $M$ for model and $P$ for prototype) with the Froude number F defined as

$$
\mathrm{F}=V /(g L)^{1 / 2}
$$

In Eq. (1) $V$ is a characteristic velocity, typically the mean flow velocity, $L$ a characteristic length, typically the water depth, and $g$ is the gravitational acceleration. The Froude number is the square root of the ratio of inertial force $\rho L^{2} V^{2}$ to gravity force $\rho L^{3} g$, with the fluid density $\rho$, such that the interplay of inertial and gravity forces is correctly represented in a Froude model. Other force ratios, such as the Reynolds number

$$
\mathrm{R}=L V / v,
$$

which represents the ratio of inertial force to viscous force with kinematic viscosity $v$, and the Weber number (inertial force to surface tension force) may be represented incorrectly in a Froude model. This may result in significant scale effects (Heller, 2011; Hughes, 1993; Le Méhauté, 1990). 
Different strategies to deal with scale effects in Froude models were reviewed by Heller (2011) including avoidance, compensation and correction. In order to avoid scale effects, an a priori defined limiting R, Weber number and others have to be exceeded, as shown for landslide-tsunamis in Heller, Hager, and Minor (2008), for dike breaching in Schmocker and Hager (2009) and for impulse wave run-up and run-over in Fuchs and Hager (2012). However, why can significant scale effects be excluded with a limiting $\mathrm{R}$ ?

This article aims to address this question and to support Froude modelling of phenomena where both the Froude and Reynolds number $\mathrm{R}$ are a priori relevant. Two concepts are identified under which R scale effects may be excluded, namely (i) Self-Similarity (SS) and (ii) R Invariance (RI). These two concepts are reviewed and illustrated with a wide range of examples. This is expected to assist the design and improve the reliability of many Froude model studies in hydraulic engineering for phenomena where inertial, gravity and viscous forces are a priori relevant: hydraulic jumps, highvelocity open channel flows, landslide-tsunami generation, wave breaking, wakes in rivers and waves, sediment transport, dike breaching, plumes and jets entering rivers and waves, turbulent flows, entrainment, and others.

\subsection{SS and RI}

SS, self-preservation, fractals, dimensional analysis, dynamic similarity, universality, symmetry, scaling laws, RI, scale invariance and insignificant scale effects are all interrelated phenomena (Cantwell, 2002; Dodds \& Rothman, 2000; Ercan, Kavvas, \& Haltas, 2014; Foss, Panton, and Yarin, 2007). The interrelation of the most relevant concepts for this article, namely SS, RI and scale invariance (no source of scale effects), are addressed in the following sections. These sections show that the two concepts SS and RI both explain why significant $R$ scale effects can be excluded with a limiting R. However, the physical background of the two concepts and how an insignificant scale effects condition is approached are different such that SS and RI are addressed individually herein.

\section{SS}

The concept of SS in mathematical physics can be traced back to Fourier (1822), where it was applied to heat conduction. A time-developing (and/or spatial) phenomenon is called self-similar if the spatial distribution of its properties at various different instances in time (and/or spatial locations) is obtained from one another by a similarity transformation (Barenblatt, 1996; Cantwell, 2002; Chanson \& Carosi, 2007; George, 1989; Gratton, 1991; Pope, 2000). As a consequence, self-similar profiles of velocity (or any other quantity) can be brought into congruence by simple scale factors which depend on only one of the variables such as location $x$ or time $t$. A further consequence for self-similar flows is that there exist solutions to its dynamical equations and boundary conditions for which, throughout its evolution, all terms (of dynamic significance) show the same relative value at the same relative location (George, 1989). 
SS can be observed, at least in the sense of an idealised asymptotic condition, in many phenomena in nature and everyday life. Figure 1 shows some examples including the geometry of Romanesco broccoli, fern and river networks. Many further phenomena were described as self-similar including the geometry of the coastline (Mandelbrot, 1983), laws in finance (Drożdż, Grümmer, Ruf, \& Speth, 2003), the size distribution of turbidite deposits (Doods \& Rothman, 2000) and most physical laws with respect to a space and time translation (Cantwell, 2002; Frisch, 1995; Wang, 1998). It is argued herein that SS is a highly useful feature in hydraulic engineering as it may reveal universal features valid for all the spatial and temporal ranges observed in fluid flows, both within a particular phenomenon and also between a phenomenon and its model at reduced scale (note that scale refers to spatial scale hereafter, if not otherwise stated).

The identification of self-similar fluid flow features is desirable due to the following interrelated key reasons (Barenblatt, 1996; Foss et al., 2007; Gratton, 1991):

(i) universal applicability, independent of the instance in time and/or spatial location,

(ii) simple computation as self-similar flows are commonly based on an Ordinary Differential Equation (ODE) rather than a Partial Differential Equation (PDE),

(iii) requirement of a reduced volume of experimental work and/or simplified data processing,

(iv) collapse of data points to a single curve or surface, and

(v) scale invariance often applies such that small and cost efficient models may be used.

This article focuses on the large class of self-similar flow features observed at large R. Self-similar phenomena observed in laminar flows and in non-fluid phenomena are therefore excluded hereafter, as only SS observed at large R helps to understand why significant scale effects can be excluded with a limiting R. Many turbulent flow features are self-similar and this concept is particularly popular in environmental and geophysical fluid dynamics at sufficient high $\mathrm{R}$ to describe wakes (Ali \& Abid, 2014; Cantwell, 2002; George, 1989; Nedić, Vassilicos, \& Ganapathisubramani, 2013; Redford, Castro, \& Coleman, 2012; Sreenivasan, 1981; Uberoi \& Freymuth, 1970), jets (Carazzo, Kaminski, \& Tait, 2006; Craske, Debugne, \& van Reeuwijk, 2015; Foss et al., 2007; George, 1989; Gutmark \& Wygnanski, 1976; Hussein, Capp, \& George, 1994; Pope, 2000), plumes (Carazzo et al., 2006; Foss et al., 2007; Morton, 1971), shear-driven entrainment (Jonker, van Reeuwijk, Sullivan, \& Patton, 2013; Kundu, 1981), mixing layers (Champagne, Pao, \& Wygnanski, 1976) and homogeneous isotropic turbulent flows (Batchelor, 1953; Cantwell, 2002; Kolmogorov, 1941; Pope, 2000). The concept of SS was also applied to core hydraulic engineering phenomena such as turbulent free surface flows including the distributions of void fraction, bubble count rate, interfacial velocity and turbulence level (Chanson \& Carosi, 2007).

\section{$R I$ and its relation to $S S$}

$\mathrm{RI}$ is a main reason as to why some phenomena in short, highly turbulent flows (e.g. hydraulic jumps, breaking waves, landslide-tsunami generation) can be modelled with Froude similarity. This may not 
be intuitive, given that Froude similarity considers the interplay of inertial and gravity forces only, which is most applicable to free surface flows where viscous effects are negligible (e.g. deep-water waves, flows over weirs).

RI refers herein to idealised fluid conditions which are asymptotic approached with increasing $R$, i.e. the conditions become $R$ invariant because $R$ increases. This is illustrated with a well-known example in hydraulic engineering, namely pipe flow energy head losses expressed in the Moody diagram (Massey, 1989). The Moody diagram provides the Darcy-Weisbach friction factor as a function of the relative pipe roughness and $\mathrm{R}$, defined with the pipe diameter $d$ and the mean flow velocity. The friction factor shown in the Moody diagram heavily depends on $\mathrm{R}$ in the laminar and transition regime, whilst it is $\mathrm{R}$ invariant in the fully turbulent regime. The friction factor for a given relative roughness is therefore the same at small scale (small $d$ and $\mathrm{R}$ ) and large scale (large $d$ and $\mathrm{R}$ ), as long as $R$ is large enough to ensure fully turbulent flow. This example shows that an $R$ invariant phenomenon directly implies scale invariance (involves no scale effects).

The technical literature includes a number of hints which may help to understand RI. Firstly, $R \rightarrow$ $\infty$ corresponds to $v \rightarrow 0$, such that the effect of the viscosity becomes negligible for high $\mathrm{R}$ flows. This is related to the observation that fluid flows around cylinders at low $\mathrm{R}$ show a number of symmetries. An object is symmetrical if one can subject it to a certain operation and it appears exactly the same after the operation (Cantwell, 2002). These symmetries start to vanish with increasing R, but some of them are restored in a statistical sense far from the boundaries for fully developed turbulence (Frisch, 1995). Further, $\mathrm{R} \rightarrow \infty$ also corresponds to large scale motions ( $L \rightarrow \infty, V \rightarrow \infty)$, such that RI can be achieved with a large model size. This may be the reason why many studies aim to exclude significant scale effects with a limiting scale factor $\lambda=L_{P} / L_{M}$ rather than with the more sound criterion of a limiting R (Heller, 2011).

Frisch (1995) discusses symmetries applying to the Navier-Stokes Equations (NSEs). For an incompressible fluid under spatial periodic boundary conditions, there exists a scale symmetry described by

$$
t, \boldsymbol{r}, \boldsymbol{v} \rightarrow \lambda^{1-m} t, \lambda \boldsymbol{r}, \lambda^{m} \boldsymbol{v} \quad \text { with } \lambda \in \mathfrak{R}_{+}, m \in \mathfrak{R} \text { and } v=0
$$

In Eq. (3) $\boldsymbol{r}=(x, y, z)$ is the position vector and $\boldsymbol{v}$ the velocity vector. Equation (3) applied to the NSEs multiplies all terms by $\lambda^{2 m-1}$, apart from the viscous term which is multiplied by $\lambda^{m-2}$. Equation (3) for $v \neq 0$ would only apply for a scaling exponent $m=-1$, resulting in Reynolds similarity with $\mathrm{R}_{M}=\mathrm{R}_{P}$ (Frisch, 1995). It follows that scale invariance for this example applies for any real number $m$ only if $v$ $=0(R=\infty)$. This indicates that the NSEs support asymptotic conditions $(R \rightarrow \infty)$ for which scale invariance is achieved. Note also that the value $m=1 / 2$ in Eq. (3) corresponds to Froude scaling.

Table 1 summarises the similarities and differences between SS and RI. Whilst SS may be observed for flows at any $\mathrm{R}$, the additional assumption of a high $\mathrm{R}$ is used for the large class of self- 
similar flows addressed herein to exclude the dependence on the viscosity. RI directly implies scale invariance, but this is not necessarily the case for a self-similar feature as a phenomenon may be selfsimilar relative to a time or velocity scale, rather than a length scale. Both SS and RI may not be reached until the initial conditions are over-come. Further, both concepts may be obstructed in vicinity of solid boundaries as $\mathrm{R}$ is locally too small to achieve RI and the solid boundary implies a dependence on rather than an independence of a length scale, preventing SS (Table 1). Also, an R invariant feature is directly approached with increasing $\mathrm{R}$, which is not necessarily the case for SS; whilst for the class of self-similar features addressed herein the collapse of data points to a single curve or surface is still expected with an even larger $R$, scale effects do not necessarily further reduce with increasing $R$. The two concepts are interrelated as both result in a reduced number of independent variables in a phenomenon. The most relevant similarity in the context of Froude modelling is, however, that both concepts reflect idealised asymptotic conditions which are useful in excluding significant scale effects in Froude models.

\subsection{Aims and content}

This article aims to improve the reliability of Froude modelling in fluid flows where both the Froude number and $R$ are a priori relevant. The article reviews various model strategies using SS and RI in the context of Froude modelling with illustrations of where and when these idealised asymptotic conditions occur. Section 2 provides the theoretical base of SS. Self-similar phenomena at large $R$ are reviewed in Section 3.1 and $R$ invariant phenomena are addressed in Section 3.2. Section 4 describes over-shadowing along with other limitations of the reviewed concepts in ruling out significant $R$ scale effects. The most relevant findings are summarised in the Conclusions.

\section{Theoretical base of SS}

SS is based on symmetry analysis, where an object is symmetrical if one can subject it to a certain operation and it appears exactly the same after the operation. The object is then said to be invariant with respect to the given operation (Cantwell, 2002). This article focuses on fluid features (objects), e.g. the mean velocity profile, which appear the same after applying a stretching operation in all three coordinate directions, such that the mean velocity profile is scale invariant. An important transformation in symmetry analysis is the one-parameter Lie group transformation, applied in a study reviewed in Section 3.1.

Hydrodynamics problems are generally described by PDEs, implying that their solutions depend on several variables such as location and/or time. These PDEs are commonly nonlinear and reduce to linear equations in certain simple cases. The solution of such linear and nonlinear PDEs is a much more complicated task than that of ODEs where functions of a single variable are sought. If a PDE is two-dimensional or axisymmetric, it will reduce to an ODE when a length scale (or possibly a time or 
velocity scale) is excluded. This was particularly important in the pre-computer era (Gratton, 1991). A first sign of the existence of SS is thus if a PDE reduces to an ODE (Cantwell, 2002; Foss et al., 2007).

SS is introduced more formally with a mathematical physics problem in two independent variables, namely radial position $r$ and time $t$, requiring the solution of PDEs. In this problem, SS means that variable scales of a parameter of a reference (subscript 0 ) value $\boldsymbol{u}_{0}(t)$ at reference position $r_{0}(t)$ can be chosen such that in the new scales the properties of the phenomenon can be expressed by functions of one SS variable $\eta$ as

$$
\boldsymbol{u}=\boldsymbol{u}_{0}(t) \mathrm{F}(\eta) \quad \text { with } \quad \eta=r / r_{0}(t)
$$

The solution of this problem is thus reduced to the solution of a system of ODEs for the vectorfunction $\mathrm{F}(\eta)$ (Barenblatt, 1996). Equation (4) indicates that the scaled parameter $\boldsymbol{u}$ as a function of $\eta$ depends only on one variable, namely $t$. Examples with similar features are shown in Figs 4 to 7, as discussed below.

Once recognised, SS can readily be found analytically or numerically, with Foss et al. (2007), George (1989), Gratton (1991) and Pope (2000) providing a selection of examples. Here the example of vortex diffusion of Foss et al. (2007) is presented based on Fig. 2a. This example does not necessarily rely on a high $R$, in contrast to all other examples reviewed in Section 3.1 and Table 2. Nevertheless, it is a simple illustration of how a self-similar solution can be found. The vorticity transport equation is obtained by applying the curl operator to the NSEs resulting for planar motion in

$$
\frac{\partial \omega}{\partial t}=\frac{v}{r} \frac{\partial}{\partial r}\left(r \frac{\partial \omega}{\partial r}\right)
$$

Equation (5) describes an irrotational (free) vortex with vorticity $\omega=$ curlv, with $\boldsymbol{v}$ as the velocity vector and $r$ as the radial coordinate. It is assumed that the flow at time $t$ depends on $r$ only and is circular symmetric. The strength of the vorticity is characterised by the initial (subscript 0 ) circulation $\Gamma_{0}$. For an initially pointwise vortex the corresponding azimuthal (subscript $\theta$ ) velocity at $t=0$ is

$$
V_{\theta}=\Gamma_{0} /(2 \pi r) .
$$

As no cross-sectional radius is given in the present vortex (idealised as a pointwise vortex) an arbitrary length scale $L$ is taken for the following derivation. Dimensional considerations result in the time scale $L^{2} / v$ and corresponding vorticity scale $\Gamma_{0} / L^{2}$. Any solution of Eq. (5) should have the dimensionless (subscript dim) form $\omega_{d i m}=\mathrm{f}\left(t_{d i m}, r_{d i m}\right)$, with $\omega_{d i m}=\omega /\left(\Gamma_{0} / L^{2}\right), t_{d i m}=t /\left(L^{2} / v\right)$ and $r_{d i m}=r / L$. This yields the dimensional vorticity

$$
\omega=\left(\Gamma_{0} / L^{2}\right) \mathrm{f}\left(t_{d i m}, r_{d i m}\right)
$$


SS requires that the final result is independent of the arbitrary length scale $L$. This is satisfied by a dimensionless function $\mathrm{f}$ depending only on $t_{\text {dim }}$ of the form

$$
\mathrm{f}\left(t_{d i m}, r_{\text {dim }}\right)=\left(1 / t_{\text {dim }}\right) \mathrm{F}_{1}(\eta) \quad \text { with } \quad \eta=r_{\text {dim }} / t_{\text {dim }}{ }^{1 / 2}
$$

and thus

$$
\omega=\left(\Gamma_{0} / v t\right) \mathrm{F}_{1}(\eta) \quad \text { with } \quad \eta=r /(v t)^{1 / 2} .
$$

Equation (9) of this problem corresponds to the general Eq. (4). The function $F_{1}(\eta)$ can be found by substituting Eq. (8) into Eq. (5) and solving the resulting ODE with the initial condition $\omega=0$ for $r>0$ and boundary condition $\omega \rightarrow 0$ for $r \rightarrow \infty$ (the limit used in this example is $r / L \rightarrow \infty$ ), resulting in

$$
\mathrm{F}_{1}(\eta)=\frac{1}{4 \pi} \exp \left(-\frac{r^{2}}{4 v t}\right)
$$

The corresponding azimuthal velocity field becomes

$$
V_{\theta}=\frac{\Gamma_{0}}{2 \pi r}\left[1-\exp \left(-\frac{r^{2}}{4 v t}\right)\right]
$$

The vorticity field is self-similar as

$$
\frac{\omega}{\Gamma_{0} /(v t)}=\mathrm{F}_{1}(\eta)
$$

Equation (12) expresses the vorticity magnitude at a particular location and time via its magnitude at any other location and time, while also excluding any length scale $L$. Note that this example assumes idealised conditions including an initially pointwise vortex. A real vortex, as shown in Fig. $2 b$, has a finite core which may be described with solid-state rotation.

\section{Phenomena involving SS and RI}

\subsection{SS phenomena at large $R$}

Figure 3 shows examples of self-similar features at large $R$. This includes a turbulent wake behind an aerofoil (Fig. 3a), a volcanic plume (Fig. 3b), turbulent air-water mixture on a chute (Fig. 3c) and suspended sediment transport in a river (Fig. 3d). Table 2 summarises features which are self-similar involving a large $\mathrm{R}$ and the conditions under which they are observed. Self-similarities of the phenomena shown in Fig. 3 are individually addressed hereafter.

\section{Wakes}


Turbulent wakes are observed downstream of many structures in hydro- or aerodynamics, including bridge piers, aerofoils (Fig. 3a) and risers. Many of these wake generators are observed in free surface flows such as open channels, rivers and waves, which are commonly modelled with Froude similarity. Turbulent wakes downstream of different objects were investigated by Nedić et al. (2013), Pope (2000), Redford et al. (2012), Uberoi and Freymuth (1970) and Wygnanski, Champagne, and Marasli (1986).

Wygnanski et al. (1986) investigated plane (2D) wakes downstream of different wake generators, namely circular cylinders, a symmetrical aerofoil, a flat plate and screens with 30, 45, 70 and 100\% of solidity located in a wind tunnel. Their tests were conducted at flow speeds of 2 to $35 \mathrm{~m} / \mathrm{s}$ and $R$, defined with the free stream velocity $u_{\infty}$ and the thickness of wake generator in cross-flow direction $\Delta y$, was varied between 1,360 and 6,500. Typical velocity defects in the centre of the wake were 0.03 $0.15 u_{\infty}$ and measurements were taken with hot-wire probes between 100 and 2,000 momentum thicknesses $\theta=C_{D} \Delta y / 2$, with $C_{D}$ as the drag coefficient. The flow in the wake at sufficient high $\mathrm{R}$ is expected to be self-similar, described by a single velocity scale $\Delta u_{\mathrm{c}}$ (the velocity defect on the centreline (subscript $c$ )) and length scale $L_{c}$ (the distance to the cross-flow position $y$ where the velocity defect is $0.5 \Delta u_{\mathrm{c}}$ ).

Figure 4 shows data presented by Wygnanski et al. (1986). The normalised mean velocity defect profiles for a 30\% solidity screen in Fig. 4a collapse on an exponential function (point (iv) in Section 1.2). Such self-similar features in a wake may only be observed at a particular spacing downstream of the wake generator (e.g. for $200<\left(x-x_{0}\right) / 2 \theta<700$ in Fig. 4a, with $x_{0}$ as the virtual origin). SS may not be observed in close proximity of the wake generator due to the initial conditions, nor further downstream as the wake effects are smoothed out and over-shadowed by background turbulence. Figure $4 \mathrm{~b}$ compares the normalised Reynolds stresses for an aerofoil, a 70\% solidity screen and a solid strip. The data are approximately self-similar for each individual wake generator; however, the overall functions describing the data are clearly not universal as they are distinct for each individual wake generator. Whether the velocity and length scales would become universal functions of $x / \theta$ at extremely large values of $x / \theta$, similar as for turbulent jets (Carazzo et al., 2006), remained open in the study of Wygnanski et al. (1986).

More research about the potential existence of a universal self-similar regime downstream of axisymmetric wakes, irrespective of the initial conditions, was conducted by Redford et al. (2012). They studied the wakes generated by two different initial conditions (spherical like wake generators of diameter $d$ ), under constant net momentum defect (drag), with Direct Numerical Simulations (DNSs). The two initial conditions were (a) a series of slightly perturbed vortex rings and (b) low-level random-phase velocity fluctuations added to the mean-velocity profile taken from (a). The mean wake $\mathrm{R}$ was $\Delta u_{c}(x=0)\left[\Delta Q /\left(2 \pi \Delta u_{c}(x=0)\right)\right]^{1 / 2} / v=2,171$ for both conditions (a) and (b), with the velocity defect on the centreline $\Delta u_{c}$ and the volume-flux defect $\Delta Q$. SS was identified for the mean velocity 
defect profile in both cases, although they are only universal for a distance $x>5000 d$. In other words, Redford et al. (2012) revealed that a universal self-similar regime for the mean velocity defect profile exists, irrespective of the initial conditions. However, given that this universal self-similar regime is observed for extremely large distances from the wake generator, this regime may not appear in flows of practical interest or may be over-shadowed by background turbulence (Section 4).

\section{Jets and plumes}

SS is widespread in jet and plume investigations as it allows for a simple parameterisation (Hussein et al., 1994; Pope, 2000). Plumes arise from smoke, effluent from submerged pollution outlets, nuclear accidents, seafloor hydrothermal vents, convection in clouds and explosive volcanic eruptions (Fig. 3b) and are dominated by buoyancy with negligible momentum flux at the source. Jets include water jet fountains, water cannons for firefighting or jet packs dominated by momentum and showing negligible buoyancy flux at the source.

Complete SS in a jet and plume assumes that the profiles of mean vertical velocity and mean buoyancy force in horizontal sections are similar at all heights $z / d$, with $d$ as the source diameter. Under self-similar conditions a solution to the averaged equations of the form $u=u_{0}(x) \mathrm{F}_{2}(\eta)$ for the mean velocity profiles is sought, in analogy to Eq. (4). This involves a reference velocity $u_{0}$ and the SS variable $\eta=r /\left(x-x_{0}\right)$ with the radial coordinate $r$, the distance from the source $x$ and the virtual origin $x_{0}$. Hussein et al. (1994) conducted detailed measurements in an axisymmetric turbulent air jet at $\mathrm{R}=$ 100,000 with hot-wire techniques and Laser-Doppler Anemometry. The mean velocity profile of the jet is shown in Fig. 5 where the data collapse on a curve for measurements taken beyond $30 \mathrm{~d}$. If the function of this curve is known a priori of an experiment, then only one velocity value at one point is required to describe the jet in the entire self-similar regime, enormously reducing the volume of experimental work (point (iii) in Section 1.2).

\section{Shear-driven entrainment}

Shear-driven entrainment is particularly relevant for the deepening of oceanic boundary layers due to surface winds or wind-driven atmospheric boundary layers. An example in hydraulic engineering is the bottom boundary layer development on spillways, although in this case SS from the boundary layer may be over-shadowed by background turbulence (Section 4).

Jonker et al. (2013) conducted a DNS to investigate the deepening of a shear-driven turbulent layer penetrating into a linearly stratified quiescence fluid. Their simulations are based on the Boussinesq approximation (small density differences) of the NSEs. Jonker et al. (2013) conducted six tests involving two shear velocities $u^{*}=1$ and $3.86 \mathrm{~cm} / \mathrm{s}$ and three buoyancy frequencies $N=1.5^{1 / 2}, 3.75^{1 / 2}$ and $7.5^{1 / 2} \mathrm{~Hz}$ where $N=(\partial b / \partial z)^{1 / 2}$ with vertical coordinate $z$, buoyancy $b=-g\left(\rho-\rho_{0}\right) / \rho_{0}$ and local $\rho$ 
and reference density $\rho_{0}$. The Prandtl number was unity for all experiments and the buoyancy $R=$ $u^{* 2} /(N v)$ was varied from $36-1,214$.

Figure 6 shows some results of Jonker et al. (2013) and illustrates how self-similar data collapse in dimensionless form (point (iv) in Section 1.2). Figure 6a shows the boundary layer growth of the shear-driven boundary layer versus time and Fig. $6 \mathrm{~b}$ shows the collapse of the same data on a straight line (power law) in dimensionless form using the reference length scale $u^{*} / N$ and the reference time scale 1/N. The results in Fig. $6 \mathrm{~b}$ are $\mathrm{R}$ invariant and reveal a growth law $h \sim t^{-1 / 2}$ for the mixed boundary layer depth. This growth law was confirmed with a SS analysis (Kundu, 1981) by Jonker et al. (2013).

\section{High-velocity open channel flows}

High-velocity open channel flows are a core topic of hydraulic engineering, typically being modelled with Froude similarity. These flows are observed on hydraulic structures such as spillways and chutes (Fig. 3c). Chanson and Carosi (2007) investigated high-velocity turbulent open channel air-water skimming flows on a stepped chute and suggested several self-similar features. A broad-crested weir was built in a $1 \mathrm{~m}$ wide and $3.2 \mathrm{~m}$ long flume followed by ten identical $0.10 \mathrm{~m}$ high and $0.25 \mathrm{~m}$ long steps. The flow features were measured with single- and dual-tip conductivity probes under variation of the discharge $Q$ and the critical flow depth $h_{c}$. The physical Froude model tests were conducted in the fully turbulent regime at $R=380,000$ to 710,000 , involving the hydraulic diameter and the mean flow velocity in $R$.

Chanson and Carosi (2007) suggest self-similar processes for various flow features including the distributions of void fraction, bubble count rate, interfacial velocity and turbulence level. All of these processes should be scale invariant and useful, as a first approximation, to characterise the air-water flow field on similar stepped spillway structures at different scales. Perhaps the most characteristic self-similar feature observed by Chanson and Carosi (2007) is shown in Fig. 7, namely the dimensionless void fraction distribution $C(\chi)$.

\section{Sediment transport}

Sediment transport is relevant in areas such as fluvial hydraulics (Fig. 3d) and coastal engineering. RI in sediment transport may be observed in the Shields diagram (Shields, 1936) where the critical Shields stress becomes independent of the boundary R, defined with the grain diameter $d_{g}$ and the shear velocity $u^{*}$, for approximately $\mathrm{R}>400$.

Carr, Ercan, and Kavvas (2015) investigated SS and scale invariance in one-dimensional (1D) unsteady suspended sediment transport (Fig. 3d). They applied the one-parameter Lie group of point scaling transformation (Cantwell, 2002) to the governing equations (Saint-Venant, unsteady 1D suspended sediment transport) and to the initial and boundary conditions. This resulted in a set of 
scaling laws, which were used to transform the parameters from the full scale (or space) to the model scale and vice versa. Note that the Lie group scaling itself does not introduce any limitations, meaning that the solutions are expected to be symmetrical (self-similar) under the applied stretching operations (transformation) if the governing equations and initial and boundary conditions correctly capture the physics of the problem (Cantwell, 2002). A key advantage of the Lie group scaling is that, in contrast to traditional Froude modelling in sediment transport (Kamphuis, 1974), the transformed parameters require no scaling of the sediment diameter and of the density, such that $\mathrm{R}_{M}=\mathrm{R}_{P}$.

Carr et al. (2015) applied the derived scaling laws to scale the geometry and initial conditions of a test case modelled with a 1D channel network model. The grain Reynolds number was in the range 10 $\leq \mathrm{R} \leq 400$. Figure 8 shows the suspended sediment concentration calculated at full scale (prototype) and the corresponding data simulated at reduced scale and up-scaled based on the Lie group scaling (Case 2L), resulting in an excellent agreement (SS). Carr et al. (2015) modelled the same case with traditional Froude modelling (vertically distorted by a factor of 14.14 and scaled sediment density) with the up-scaled results also included in Fig. 8 (Case 2F). Scale effects are observed, essentially due to deviating $R$ between the prototype and Case $2 \mathrm{~F}$. In contrast, $R$ in Case $2 \mathrm{~L}$ is identical to that in the prototype.

\section{Homogeneous isotropic turbulence and locally homogeneous isotropic turbulence}

In homogeneous isotropic turbulence the average properties of a random motion are independent of the position and direction of the axes of reference in the fluid (Batchelor, 1953). The following shows that under these conditions, the statistics of the longitudinal velocity differences between two points is self-similar in a particular range. This is different to the previous examples of Section 3.1 where a solution of a particular problem was found to be self-similar rather than the statistics of flow features. It is further worth noting that the hereafter presented features of turbulent fluid flows can be derived without using the NSEs.

An important concept in fully turbulent flows is the energy cascade model (Kolmogorov, 1941; Richardson, 1922). In this model the turbulence is composed of eddies of different sizes as schematically shown in Fig. 9. An eddy is a turbulent motion, localised within a region of size $l$ with a characteristic velocity $V(l)$. Kinetic energy enters the turbulent flow at the largest or initial scales of motion $l_{0}$, strongly influenced by the geometry of the flow. These large eddies are unstable and break up, transferring their energy to somewhat smaller eddies, which undergo a similar process and transfer their energy to even smaller eddies. This process continues until the Reynolds number $\mathrm{R}(l)=l V(l) / v$ is sufficiently small $(\approx 1)$, such that the eddy motion is stable, and molecular viscosity is effective in dissipating the kinetic energy (Batchelor, 1953; Pope, 2000).

Kolmogorov (1941) postulated that at sufficient high $\mathrm{R}$, the small-scale turbulent motions are statistically isotropic (hypothesis of local isotropy). This implies that the statistics of the small-scale motions are universal (self-similar) in the sense that they do not remember the geometry of the large 
eddies. This size range is referred to as the universal equilibrium range, given by sizes $<l_{E I}$ (subscript $E I$ for energy-inertial) as shown in Fig. 9. Kolmogorov further postulated in his $1^{\text {st }}$ similarity hypothesis that the statistics of the small-scale motions $\left(l<l_{E I}\right)$ have a universal form that is uniquely determined by the kinematic viscosity $v\left(\mathrm{~m}^{2} \mathrm{~s}^{-1}\right)$ and the dissipation rate $\varepsilon\left(\mathrm{m}^{2} \mathrm{~s}^{-3}\right)$. Simple dimensional considerations result in the Kolmogorov (subscript $K$ ) microscale $\eta_{K}=\left(v^{3} / \varepsilon\right)^{1 / 4}$ determining the size of the smallest eddies. The universal equilibrium range may be further separated into the inertial subrange $l_{I D} \leq l \leq l_{E I}$ (subscript $I D$ for inertial-dissipation), where the statistics of the motions of eddies is uniquely determined by $\varepsilon\left(2^{\text {nd }}\right.$ similarity hypothesis), and the dissipation range $l<l_{I D}$, where the statistics of eddies depends on both $\varepsilon$ and $v$ (Fig. 9).

The energy spectrum $E(\kappa)$ is a common way to investigate how the turbulent kinetic energy is distributed among the eddies of different sizes, where $\kappa=2 \pi / l$ corresponds to a wave number. A direct consequence of Kolmogorov's $1^{\text {st }}$ and $2^{\text {nd }}$ similarity hypotheses is that the energy spectra in the inertial range is given by

$$
E(\kappa)=C_{K} \varepsilon^{2 / 3} \kappa^{-5 / 3}
$$

where $C_{K}=1.5$ is a universal constant (Pope, 2000). This is the famous Kolmogorov $-5 / 3$ spectrum, which was confirmed in countless studies in the past 50 years (e.g. Champagne et al., 1976; Frisch, 1995; Parsons \& García, 1998; Pope, 2000; Uberoi \& Freymuth, 1970; Vassilicos, 2015). An example about gravity currents will be discussed in Section 3.2 (Fig. 10c).

Whether the statistics of the velocities in a turbulent flow is self-similar (universal) across a wide range of spatial scales is usually investigated by examining the Probability Distribution Function (PDF) of longitudinal velocity differences between two points of separation $l, \Delta u(l)=u(x+l)-u(x)$, where $u$ points along the line connecting the two points. SS requires that the PDFs have a functional form independent of the separation $l$, as the statistics need to be scale invariant. The confirmation of the $-5 / 3$ dependency for the inertial subrange in a phenomenon is a strong indication for SS.

\subsection{R invariant phenomena}

This section addresses some $R$ invariant phenomena. Figure 10 shows some examples for which $R$ invariant features were proposed, including a horizontal axis tidal turbine (Fig. 10a), solute transport in a chlorine contact tank (Fig. 10b) and a gravity current in the atmosphere (Fig. 10c). These three phenomena are reviewed in more detail hereafter and Table 3 summarises $R$ invariant features of various phenomena and the conditions under which they are observed. Further studies including RI are presented in Heller (2011) and are not repeated herein.

\section{Tidal energy converters}


Tidal energy is a promising marine renewable energy source. Tens of Tidal Energy Converters (TECs) are currently under research and development (Fig. 10a). Reynolds similarity would be most appropriate to model TECs, or marine hydrokinetic turbines in general, at reduced scale as the flow mainly depends on R. Froude similarity is inappropriate as free surface effects (gravity) are in most situations negligible (Bachant \& Wosnik, 2016) and as Froude modelling would result in incorrect power output and structural load. If, in addition to Reynolds similarity, the tip-speed ratio is correctly modelled, full similarity may be expected. However, Reynolds similarity assuming $\mathbf{R}_{M}=\mathbf{R}_{P}$ and $m=-$ 1 in Eq. (3) results in an unpractical scaling law $\lambda^{-1}$ for the velocity. This implies that the flow velocity in a 1: $\lambda$ scale model needs to be $\lambda$ times larger than in the prototype. Similarly, rotation per minute need to be $\lambda^{2}$ times larger in the model than in the prototype. This requires extremely high flow velocities in the model and results in secondary effects such as cavitation. Reynolds similarity is therefore highly unpractical or even impossible to achieve.

The most appropriate model strategy for a TEC depends on which parameters are of interest. A common approach is to keep the tip speed ratio constant between model and prototype and to select the (chord) Reynolds number as large as possible to be close to the $\mathrm{R}$ invariant regime (Bachant $\&$ Wosnik, 2016). Myers and Bahaj (2012), however, modelled TECs as porous actuator disks and were interested in the wake structure downstream of TEC arrays, with the correct modelling of the amount of thrust force on the fluid flow being considered most important. Consequently, they kept the thrust force coefficient constant between model and prototype and ensured that $R$ (based on the free stream velocity and water depth) was clearly larger than 2,000. This should assure RI and a similar regime as at full scale where fully turbulent conditions are expected, with $R \sim 10^{7}$. Nevertheless, both model strategies, based on the tip speed ratio and the thrust coefficient, are likely to result in significant scale effects. These uncertainties are the reason why guidelines for investigating TECs strongly recommend testing devices at several scales.

Bachant and Wosnik (2016) investigated a 3-bladed cross-flow (vertical axis) turbine with an overall diameter of $1 \mathrm{~m}$ in a $36.6 \mathrm{~m}$ long, $3.66 \mathrm{~m}$ wide and $2.44 \mathrm{~m}$ deep towing tank. The Reynolds number, defined with the turbine diameter $d$ and the tow carriage velocity (free stream velocity $u_{\infty}$ ), was increased in steps of $10^{5}$ from 0.3 to $1.3 \times 10^{6}$ to investigate RI on the mean power (subscript $P$ ) coefficient $c_{P}$ and drag coefficient. The experimental results are shown in Fig. 11 and illustrate the asymptotic approach of an $\mathrm{R}$ invariant $c_{P}$ level at high $\mathrm{R}$. The $\mathrm{R}$ invariant regime is reached at $\mathrm{R} \approx$ 800,000 , which can be achieved in water with a large $d$ and/or $u_{\infty}$ (Bachant \& Wosnik, 2016).

\section{Complete mixing in contact tanks}

A contact tank (Fig. 10b) is commonly used to disinfect drinking water prior to distribution. Teixeira and Rauen (2014) investigated the effects of scale and discharge variation on solute transport in an Lshaped chlorine contact tank, where the flow pattern is mostly fully turbulent and tends to complete 
mixing. The physical and chemical flow processes in a contact tank may depend on $F, R$ and the solute residence time, each of which implies a different scaling law for the discharge $Q$.

In order to address this challenge and to investigate dynamic similarity, Teixeira and Rauen (2014) varied both the model scale and the discharge based on Froude similarity. Their tests involved a fullscale tank $(\lambda=1)$ with prototype discharge $Q_{P}=0.21 \mathrm{~m}^{3} / \mathrm{s}$ and Froude models at scales $\lambda=8,16,24$ and 50, each tested with model discharges $0.50 Q_{M}, 0.75 Q_{M}, Q_{M}, 1.25 Q_{M}$ and $1.50 Q_{M}$ where $Q_{M}=$ $Q_{P} / \lambda^{5 / 2}$, according to Froude modelling. The corresponding R changed by more than an order of magnitude in these experiments. Saline tracer was injected at the tank inlet and measured at the outlet with a conductivity probe. Potential scale effects at the outlet were assessed with the residence time distribution curves of the different models, where discrepancies were assessed with the curve area discrepancy index. Figure 12a shows this index for the tests in the smallest three scale models with $\lambda=$ 16, 24 and 50 compared with the model with $\lambda=8$. As the flow conditions are fully or almost fully turbulent, the curve area discrepancy index is scale and discharge invariant, such that the flow conditions are indeed in the $R$ invariant regime.

Using the same method, Teixeira and Rauen (2014) also investigated a contact tank tending to plug flow with most data in the laminar $(R<2,000)$ and transition $(2,000 \leq R \leq 4,000)$ regime where no scale invariance is expected. The results are shown in Fig. 12b. Increasing scale effects with increasing $\lambda$ are indeed observed and scale effects are significant (approximately 20\%) for the smallest scale with $\lambda=50$.

\section{Gravity currents}

Gravity, density or buoyancy currents are buoyancy driven fluid flows moving as a result of density differences primarily in the horizontal direction (Fig. 10c). The density difference is caused by temperature or by dissolved (salinity) or suspended material (turbidity currents, snow avalanches). Examples of gravity currents include thunderstorm outflows, sea-breeze fronts, river front mixing with sea water in estuaries, base surges formed from gases and solids from volcanic eruptions, snow avalanches and turbidity currents (Simpson, 1997).

One approach to investigate gravity currents with is the arrested gravity current method. Parsons and García (1998) conducted experiments to investigate (self-)similarity with this method in the set-up shown in Fig. 13a. The water level in the channel was controlled with an overflow weir and the denser fluid was fed through the bottom corner in front of this weir. Clear water was pumped into the other channel end such that it flew over the denser fluid and the weir. The channel floor was moved with a conveyor belt reaching upfront of the current head such that the quasi steady gravity current was approached by a uniform velocity profile. Parsons and García (1998) conducted tests under variation of the initial excess density difference, the current height behind the front $h_{5}$, the height of the current front $h_{f}$ and the propagation velocity of the current front $u_{1}$, corresponding to the conveyor belt 
velocity (Fig. 13a). The behaviour of the front is described by the dimensionless mixing rate $0.066 \leq$ $g^{\prime} q / u_{1}^{3} \leq 0.226$, the height ratio $0.070 \leq h_{5} / h_{1} \leq 0.114$ and the Reynolds number $381 \leq h_{5}\left(g^{\prime} q\right)^{1 / 3} / v \leq$ 2,012 defined with $h_{5}$ as the characteristic length and the cube root of the buoyancy flux into the current front $\left(g^{\prime} q\right)^{1 / 3}$ as the characteristic velocity, respectively. The parameter $h_{1}$ is the clear water depth, $g^{\prime}=g\left(\left(\rho-\rho_{0}\right) / \rho_{0}\right)$ the reduced gravity and $q$ the specific discharge. The front mixing was quantified with a conductivity probe sampling at $800 \mathrm{~Hz}$ and visualised with Laser-induced fluorescence experiments.

Parsons and García (1998) showed that the data collapse on a single curve in the form of the power spectra for $\mathrm{R} \geq 1,000$. Tests conducted at lower $\mathrm{R}$ deviate considerable from this self-similar statistical behaviour. Figure $13 \mathrm{~b}$ compares the dimensional power spectra $G_{x x}(f)$ of a test with $\mathrm{R}=600$ to a selfsimilar test with $R=1,108$. Considerable differences are observed since the production of turbulent energy is being affected by viscosity in the smaller $\mathrm{R}$ flow suppressing mixing. In many previous studies $\mathrm{R}$ was smaller than 1,000 , resulting in significant scale effects (Parsons \& García, 1998). The authors emphasise that their results only apply to the most energetic area of the current front, such that other areas may result in scale effects even for $R \geq 1,000$. Note that the data in Fig. 13b in the inertial subrange reasonably well follows the Kolmogorov $-5 / 3$ spectrum (Eq. 13).

\section{Over-shadowing effects}

The existence of SS does not guarantee that such a motion is actually dominant. Self-similar criteria are necessary but not sufficient conditions to explicitly observe SS (Kundu, 1981). A self-similar feature may be over-shadowed by other, more dominant effects. For example, the shear-driven entrainment experiments described in Section 3.1 reveal a sound self-similar behaviour. However, these experiments were highly idealised in the sense that no Coriolis force and other sources of background turbulence were present. Background turbulence may be more dominant than the entrainment due to the shear layer such that SS would never explicitly be observed under real conditions. This may be a main reason why the collapse of the data in Fig. 7 (turbulent air-water mixture) is considerable less sound that in Fig. 6b (entrainment in quiescence fluid). Further, SS is an idealised asymptotic condition reached after the initial conditions are over-come requiring potentially a long time or distance, such that SS may never be reached.

SS and RI are valuable concepts for Froude modelling to exclude significant R scale effects. However, other force ratios may also introduce scale effects, and they may even interfere with features a priori believed to be $\mathrm{R}$ invariant. For example, in hydraulic jumps energy dissipation is approximately correctly modelled in (generalised) Froude models (Le Méhauté, 1990). However, a hydraulic jump involves considerable air entrainment depending, amongst other parameters, on surface tension (Weber number). Surface tension effects imply that air bubble sizes are incorrectly scaled in Froude models (Heller, 2011). Entrainment of a different amount of air in relation to the full scale or entrainment of larger air bubbles may have secondary effects and indirectly introduce scale 
effects on energy dissipation. Potential scale effects due to force ratios other than $\mathrm{R}$ may be a further reason as to why SS in its purest form is observed for pure water flows such as wakes (Fig. 4) or shear-driven entrainment (Fig. 6), whilst self-similar features for two-phase flows, such as highvelocity open channel flows (Fig. 7), are considerable less sound.

It is important under which conditions SS and RI are observed in a particular study. The overviews of various self-similar and $\mathrm{R}$ invariant phenomena in Tables 2 and 3 include this information. For example, Ali and Abid (2014) found that the azimuthal velocity profiles in the wake vortex core of a rotor behave self-similar with age (time). SS in this example cannot simply be assumed for the entire phenomenon, for another parameter then for that identified or for the correct parameter but in relation to a spatial rather than a time scale. SS and RI may further only apply to a particular region in the flow; the findings of Parsons and García (1998) apply to the gravity current head only. In another region, significant $R$ scale effects may be expected. Further, phenomena involving biological or chemical processes, such as water and wastewater treatment tanks (Teixeira \& Rauen, 2014), may require a certain amount of time for the reactions or processes to take place, irrespective of whether the turbulent mixing processes are self-similar.

Despite these limitations, SS and RI are important concepts to exclude significant $R$ scale effects. These concepts, along with the various studies summarised in Tables 2 and 3, are hoped to support many future Froude model studies where inertial, gravity and viscous forces are a priori relevant.

\section{Conclusions}

This review aims to improve the reliability of Froude modelling in fluid flows where both the Froude number $\mathrm{F}$ and Reynolds number $\mathrm{R}$ are a priori relevant. Two concepts are identified which may help to exclude significant scale effects under these conditions: (i) Self-Similarity (SS) and (ii) R Invariance (RI).

A time-developing (and/or spatial) phenomenon is called self-similar if the spatial distribution of its flow properties at various different instances in time (and/or spatial locations) can be obtained from one another by a similarity transformation. The most favourable feature of self-similar data in the context of Froude modelling may be their collapse to a single curve or surface. In other words, such phenomena may be correctly modelled in Froude models (no significant source of scale effects) for phenomena which would otherwise depend on both F and R. Both phenomena SS and RI are based on symmetry analysis. RI corresponds to a negligible effect of the kinematic viscosity $(v \rightarrow 0)$ and/or to a large scale motion (characteristic length $L \rightarrow \infty$, characteristic velocity $V \rightarrow \infty$ ) and directly implies scale invariance (no source of significant scale effects). SS and RI are interrelated as both reduce the number of independent variables in a phenomenon and both reflect idealised asymptotic conditions which are useful in excluding significant $R$ scale effects in Froude models.

A wide range of examples illustrating these two concepts are reviewed: (i) irrotational vortices, wakes, jets and plumes, shear-driven entrainment, high-velocity open channel flows, sediment 
transport and homogeneous isotropic turbulence and (ii) tidal energy converters, complete mixing in contact tanks and gravity currents. Tables 2 and 3 summarise all these and further phenomena with the limitations under which SS and RI are observed, with the aim to assist physical Froude modelling.

The identification of SS does not guarantee that it is explicitly observed as it may be overshadowed by more dominant effects, such as background turbulence. Further, SS and RI are idealised asymptotic conditions. For example, to observe SS the initial conditions may have to be over-come, potentially requiring a long time or distance such that SS may never be reached in a phenomenon. In addition, excluding $R$ scale effects in Froude models leaves still room for significant scale effects from other force ratios, such as the Weber number. Despite these limitations, SS and RI are important concepts to avoid significant $\mathrm{R}$ scale effects in Froude models for phenomena such as wakes, jets and plumes. These concepts may further assist in modelling short, highly turbulent phenomena and highvelocity open channel flows. Both concepts are hoped to support many future Froude model studies.

RI was applied in many further phenomena, besides these included in Table 3, to exclude significant scale effects in Froude models (Heller, 2011). Although scale invariance does not directly imply SS, it may be possible to establish SS, at least for some of these R invariant features, in future work.

\section{Acknowledgement}

The author would like to thank Dr Maarten van Reeuwijk, for helpful discussions and critical comments on an earlier version of this article, and Matthew Kesseler for linguistic suggestions.

\section{Funding}

This work was initiated during an Imperial College London Research Fellowship (cohort 2011). 


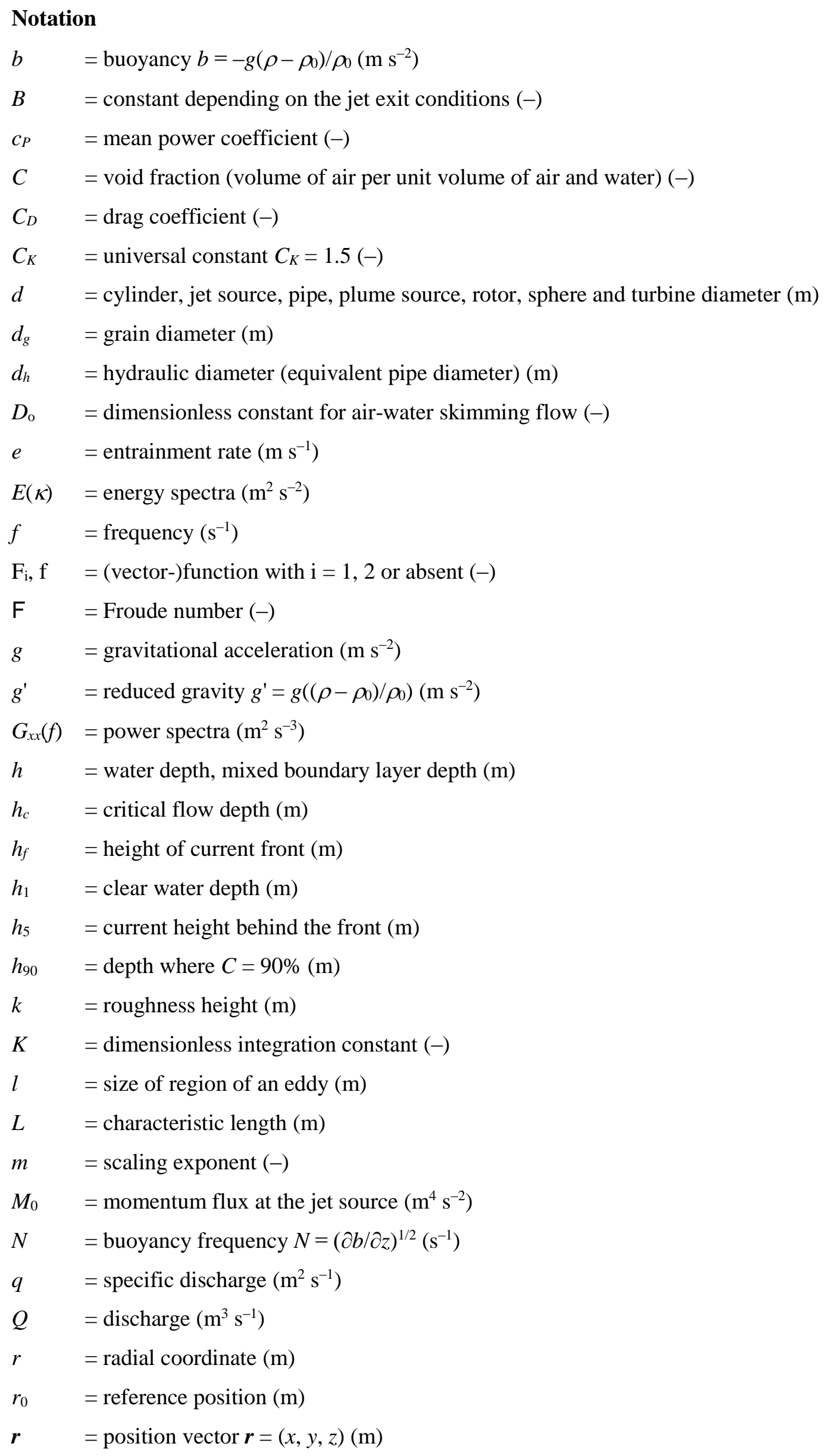




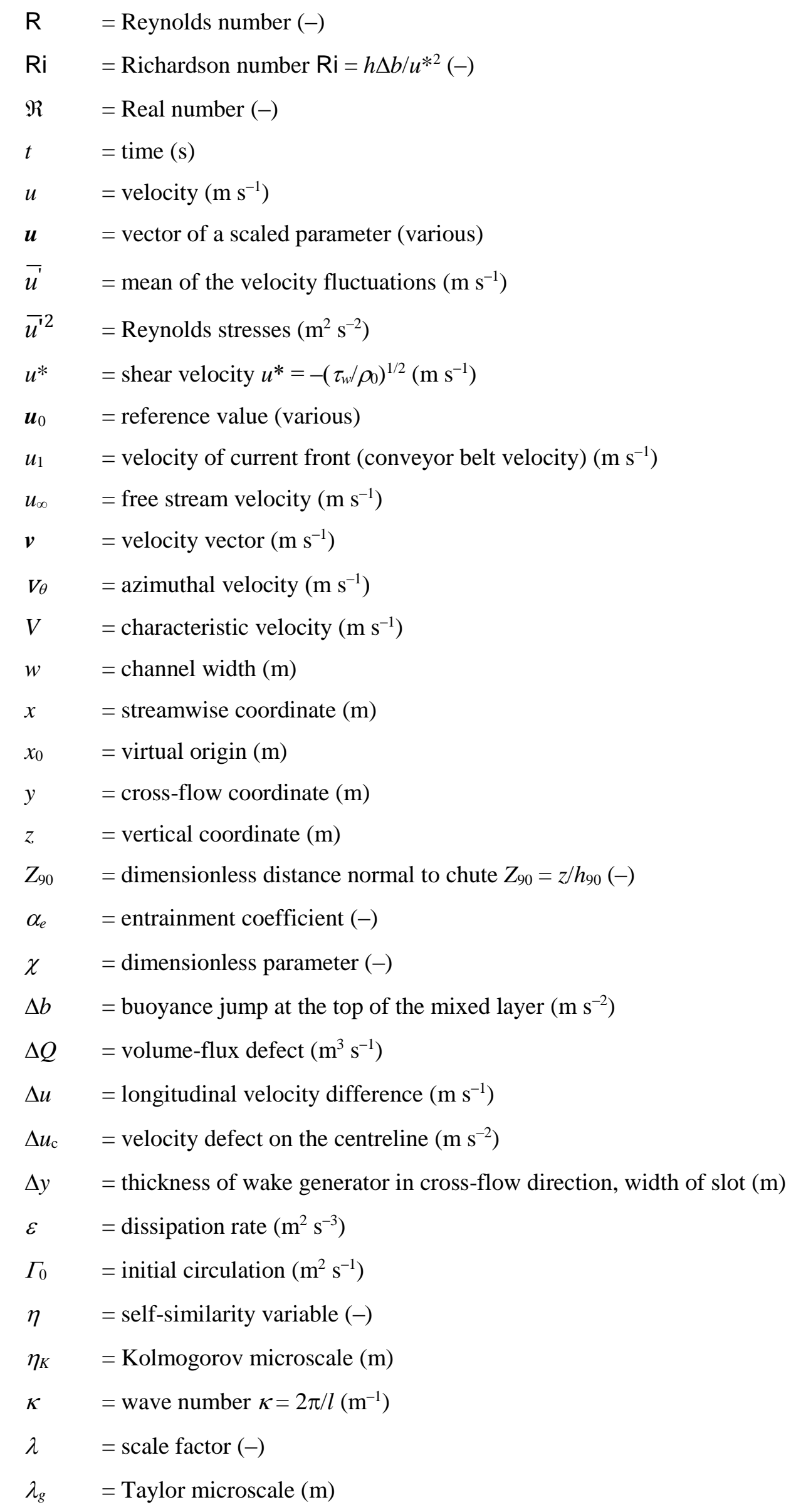




$$
\begin{array}{ll}
v & =\text { kinematic viscosity }\left(\mathrm{m}^{2} \mathrm{~s}^{-1}\right) \\
\omega & =\text { vorticity }\left(\mathrm{s}^{-1}\right) \\
\pi & =\text { numerical constant }(-) \\
\rho & =\text { fluid density }\left(\mathrm{kg} \mathrm{m}^{-3}\right) \\
\tau_{w} & =\text { shear stress }\left(\mathrm{kg} \mathrm{m}^{-1} \mathrm{~s}^{-2}\right) \\
\theta & =\text { momentum thickness }(\mathrm{m}), \text { azimuth }\left(^{\circ}\right)
\end{array}
$$

\section{Subscripts}

$$
\begin{array}{ll}
c & =\text { centreline } \\
\operatorname{dim} & =\text { dimensionless } \\
E I & =\text { energy-inertial } \\
I D & =\text { inertial-dissipation } \\
K & =\text { Kolmogorov } \\
M & =\text { model } \\
P & =\text { prototype, power } \\
\theta & =\text { azimuthal } \\
0 & =\text { initial }, \text { reference }
\end{array}
$$

\section{Abbreviations}

DNS = Direct Numerical Simulation

NSEs = Navier-Stokes Equations

ODE = Ordinary Differential Equation

PDE = Partial Differential Equation

PDF = Probability Distribution Function

RI = Reynolds number Invariance

SS = Self-Similarity

TEC = Tidal Energy Converter

$1 \mathrm{D}, 2 \mathrm{D}=$ one-dimensional, two-dimensional 


\section{References}

Ali, M., \& Abid, M. (2014). Self-similar behaviour of a rotor wake vortex core. Journal of Fluid Mechanics, 740, R1 1-12.

Bachant, P., \& Wosnik, M. (2016). Effects of Reynolds number on the energy conversion and nearwake dynamics of a high solidity vertical-axis cross-flow turbine. Energies, 9(2), 1-18.

Barenblatt, G.I. (1996). Scaling, self-similarity, and intermediate asymptotics. Cambridge: Cambridge University Press.

Baroud, C.N., Plapp, B.B., She, Z.-S., \& Swinney, H.L. (2002). Anomalous self-similarity in a turbulent rapidly rotating fluid. Physical Review Letters, 88(11), 114501-1-4.

Batchelor, G.K. (1953). The theory of homogeneous turbulence. Cambridge: Cambridge University Press.

Cantwell, B.J. (2002). Introduction to symmetry analysis. Cambridge: Cambridge University Press.

Carazzo, G., Kaminski, E., \& Tait, S. (2006). The route to self-similarity in turbulent jets and plumes. Journal of Fluid Mechanics, 547, 137-148.

Carr, K.J., Ercan, A., \& Kavvas, M.L. (2015). Scaling and self-similarity of one-dimensional unsteady suspended sediment transport with emphasis on unscaled sediment material properties. Journal of Hydraulic Engineering-ASCE, 141(5), 04015003-1-9.

Champagne, F.H., Pao, Y.H., \& Wygnanski, I.J. (1976). On the two-dimensional mixing region. Journal of Fluid Mechanics, 74(part 2), 209-250.

Chanson, H., \& Carosi, G. (2007). Turbulent time and length scale measurements in high-velocity open channel flows. Experiments in Fluids, 42(3), 385-401.

Chaplin, J.R., \& Subbiah, K. (1997). Large scale horizontal cylinder forces in waves and currents. Applied Ocean Research, 19(3-4), 211-223.

Craske, J., Debugne, A.L.R., \& van Reeuwijk, M. (2015). Shear-flow dispersion in turbulent jets. Journal of Fluid Mechanics, 781, 28-51.

Dodds, P.S., \& Rothman, D.H. (2000). Scaling, universality, and geomorphology. Annual Review of Earth and Planetary Sciences, 28, 571-610.

Drożdż, S., Grümmer, F., Ruf, F., \& Speth, J. (2003). Log-periodic self-similarity: an emerging financial law? Physica A, 324(1-2), 174-182.

Ercan, A., Kavvas, M.L., \& Haltas, I. (2014). Scaling and self-similarity in one-dimensional unsteady open channel flow. Hydrological Processes, 28(5), 2721-2737.

Foss, J.F., Panton, R., \& Yarin, A.L. (2007). Nondimensional representation of the boundary-value problem. In C. Tropea, A.L. Yarin, \& J.F. Foss (Eds.). Springer handbook of experimental fluid mechanics, Part A, Chapter 2 (pp. 33-82). Heidelberg, Germany: Springer.

Fourier, J. (1822). Theorie analytique de la chaleur [The analytical theory of heat]. Paris: Firmin Didot. 
Frisch, U. (1995). Turbulence: the legacy of A.N. Kolmogorov. Cambridge: Cambridge University Press.

Fuchs, H., \& Hager, W.H. (2012). Scale effects of impulse wave run-up and run-over. Journal of Waterway, Port, Coastal, and Ocean Engineering-ASCE, 138(4), 303-311.

George, W.K. (1989). The self-preservation of turbulent flows and its relation to initial conditions and coherent structures. In W.K. George, \& R.E.A. Arndt (Eds.). Advances in turbulence (pp. 39-73). New York: Hemisphere.

Gratton, J. (1991). Similarity and self similarity in fluid dynamics. Fundamentals of Cosmic Physics, $15,1-106$.

Gutmark, E., \& Wygnanski, I. (1976). The planar turbulent jet. Journal of Fluid Mechanics, 73(part 3), 465-495.

Heller, V. (2011). Scale effects in physical hydraulic engineering models. Journal of Hydraulic Research, 49(3), 293-306.

Heller, V., Hager, W.H., \& Minor, H.-E. (2008). Scale effects in subaerial landslide generated impulse waves. Experiments in Fluids, 44(5), 691-703.

Hughes, S.A. (1993). Physical models and laboratory techniques in coastal engineering. Advanced series on ocean engineering 7. London: World Scientific.

Hussein, H.J., Capp, S.P., \& George, W.K. (1994). Velocity measurements in a high-Reynoldsnumber, momentum-conserving, axisymmetric, turbulent jet. Journal of Fluid Mechanics, 258, 31-75.

Jonker, H.J.J., van Reeuwijk, M., Sullivan, P.P., \& Patton, E.G. (2013). On the scaling of shear-driven entrainment: a DNS study. Journal of Fluid Mechanics, 732, 150-165.

Kamphuis, J.W. (1974). Practical scaling of coastal models. Proceedings of $14^{\text {th }}$ Coastal engineering conference, 3 (pp. 2086-2101). New York: ASCE.

Kolmogorov, A.N. (1941). The local structure of turbulence in incompressible viscous fluid for very large Reynolds numbers. Doklady Akademiia Nauk SSSR, 30, 299-303 (in Russian).

Kundu, P.K. (1981). Self-similarity in stress-driven entrainment experiments. Journal of Geophysical Research, 86(C3), 1979-1988.

Le Méhauté, B. (1990). Similitude. In B. Le Méhauté, \& D.M. Hanes, (Eds.). Ocean engineering science: the sea (pp. 955-980). New York: Wiley.

Mandelbrot, B.B. (1983). The fractal geometry of nature. San Francisco: Henry Holt and Company.

Massey, B.S. (1989). Mechanics of fluids (6th ed.). London: Chapman and Hall.

Morton B.R. (1971). The choice of conservation equations for plume models. Journal of Geophysical Research, 76(30), 7409-7416.

Myers, L.E., \& Bahaj, A.S. (2012). An experimental investigation simulating flow effects in first generation marine current energy converter arrays. Renewable Energy, 37(1), 28-36.

Nedić, J., Vassilicos, J.C., \& Ganapathisubramani, B. (2013). Axisymmetric turbulent wakes with new nonequilibrium similarity scalings. Physical Review Letters, 111, 144503-1-5. 
Parsons, J.D., \& García, M.H. (1998). Similarity of gravity current fronts. Physics of Fluids, 10(12), 3209-3213.

Pope, S.B. (2000). Turbulent flows. Cambridge: Cambridge University Press.

Redford, J.A., Castro, I.P., \& Coleman, G.N. (2012). On the universality of turbulent axisymmetric wakes. Journal of Fluid Mechanics, 710, 419-452.

Richardson, L.F. (1922). Weather prediction by numerical process. Cambridge: Cambridge University Press.

Schmocker, L., \& Hager, W.H. (2009). Modelling dike breaching due to overtopping. Journal of Hydraulic Research, 47(5), 585-597.

Schultz, M.P., \& Flack, K.A. (2013). Reynolds-number scaling of turbulent channel flow. Physics of Fluids, 25, 025104-1-13.

Shields, A. (1936). Anwendung der Ähnlichkeitsmechanik und der Turbulenzforschung auf die Geschiebebewegung [Application of similarity principles and turbulence research to bed-load movement]. Mitteilungen der Preussischen Versuchsanstalt fur Wasserbau und Schiffbau, Berlin (in German).

Simpson, J.E. (1997). Gravity currents - In the environment and the laboratory. Cambridge: Cambridge University Press.

Smits, A.J., McKeon, B.J., \& Marusic, I. (2011). High-Reynolds number wall turbulence. Annual Review of Fluid Mechanics, 43, 353-375.

Sreenivasan, K.R. (1981). Approach to self-preservation in plane turbulent wakes. AIAA Journal, 19(10), 1365-1367.

Taylor, G. (1954). The dispersion of matter in turbulent flow through a pipe. Proceedings of the Royal Society of London A, 223(1155), 446-468.

Teixeira, E.C., \& Rauen, W.B. (2014). Effects of scale and discharge variation on similitude and solute transport in water treatment tanks. Journal of Environmental Engineering-ASCE, 140(1), $30-39$.

Toombes, L., \& Chanson, H. (2007). Surface waves and roughness in self-aerated supercritical flow. Environmental Fluid Mechanics, 7(3), 259-270.

Uberoi, M.S., \& Freymuth, P. (1970). Turbulent energy balance and spectra of the axisymmetric wake. Physics of Fluids, 13(9), 2205-2210.

Vassilicos, J.C. (2015). Dissipation in turbulent flows. Annual Review of Fluid Mechanics, 47, 95114.

Wang, L. (1998). Self-similarity of fluid flows. Applied Physics Letters, 73(10), 1329-1330.

Wygnanski, I., Champagne, F., \& Marasli, B. (1986). On the large-scale structures in twodimensional, small-deficit, turbulent wakes. Journal of Fluid Mechanics, 168, 31-71.

Wygnanski, I., \& Fiedler, H.E. (1969). Some measurements in the self-preserving jet. Journal of Fluid Mechanics, 38(part 3), 577-612. 
Table 1 Differences and similarities of SS and RI

\begin{tabular}{lll}
\hline Criterion & SS & RI \\
\hline Base of concept & Symmetry analysis & Symmetry analysis \\
\hline Restriction to fluid flows & No (but mainly reviewed for fluid flows herein) & Yes \\
\hline Restriction to high R flows & No (but often assumed and mainly reviewed for high & Yes \\
& R herein) & \\
\hline Correspondence to scale & Not necessarily, e.g. if observed relative to a time or & Yes \\
invariance & velocity scale rather than a length scale & \\
\hline Reduction of number of & Yes (length scale, time scale, velocity scale, etc.) & Yes (R) \\
independent variables & & \\
\hline Idealised asymptotic condition & Yes & Yes \\
\hline Dependent on initial conditions & Yes & Yes \\
\hline $\begin{array}{l}\text { Obstructed in vicinity of solid } \\
\text { boundaries }\end{array}$ & Yes & Yes \\
\hline Useful to exclude significant & Yes & Yes \\
scale effects & & \\
\hline
\end{tabular}


Table 2 Phenomena and quantities involving SS at large $\mathrm{R}$ with limitations and references

\begin{tabular}{|c|c|c|c|c|}
\hline Phenomenon & Self-similar quantity & $\begin{array}{l}\text { Investigated } \\
R \text { range } \\
\end{array}$ & Comment & Reference \\
\hline Axisymmetric jet & $\begin{array}{l}\text { Front position and spread } \\
\text { of radial integral of } \\
\text { ensemble-averaged } \\
\text { concentration of passive } \\
\text { scalar transport }\end{array}$ & $\begin{array}{l}2 M_{0}^{1 / 2} / v= \\
4,815\end{array}$ & $\begin{array}{l}\text { Ensemble average over } \\
16 \text { DNS tests; } \\
\text { negligibility of viscous } \\
\text { effects was confirmed } \\
\text { with additional tests at } \\
2 M_{0}^{1 / 2} / v=6,810\end{array}$ & $\begin{array}{l}\text { Craske et al. } \\
(2015)\end{array}$ \\
\hline Axisymmetric jet & $\begin{array}{l}\text { Mean velocity profile (Fig. } \\
\text { 5), centreline velocity and } \\
\text { many higher order } \\
\text { moments and velocities }\end{array}$ & $\begin{array}{l}d u / v= \\
100,000\end{array}$ & $\begin{array}{l}z / d>50 \text { (not everybody } \\
\text { agrees, see e.g. Carazzo } \\
\text { et al., 2006) }\end{array}$ & $\begin{array}{l}\text { Hussein et al. } \\
\text { (1994) }\end{array}$ \\
\hline $\begin{array}{l}\text { Axisymmetric } \\
\text { wake downstream } \\
\text { of a sphere }\end{array}$ & $\begin{array}{l}\text { Mean velocity defect and } \\
\text { turbulent velocity } \\
\text { fluctuation profiles }\end{array}$ & $d u_{\infty} / v=8,600$ & $\begin{array}{l}50<x / d<150, \text { data } \\
\text { collapse to a curve if } \\
\text { normalised with the } \\
\text { characteristic velocity } \\
\text { scale } u_{\infty} d^{2 / 3}\left(x-x_{0}\right)^{-2 / 3} \\
\text { and the characteristic } \\
\text { length scale }\left(x-x_{0}\right)^{1 / 3} d^{2 / 3}\end{array}$ & $\begin{array}{l}\text { Uberoi and } \\
\text { Freymuth } \\
\text { (1970) }\end{array}$ \\
\hline $\begin{array}{l}\text { Axisymmetric } \\
\text { wake downstream } \\
\text { of a spherical } \\
\text { body }\end{array}$ & $\begin{array}{l}\text { Mean velocity defect } \\
\text { profile }\end{array}$ & $\begin{array}{l}\Delta u_{\mathrm{c}}(x= \\
0)\left[\Delta Q /\left(2 \pi \Delta u_{c}(\right.\right. \\
x=0))]^{1 / 2} / v= \\
2,171\end{array}$ & $\begin{array}{l}\text { Universal SS for } x> \\
5000 d\end{array}$ & $\begin{array}{l}\text { Redford et al. } \\
\text { (2012) }\end{array}$ \\
\hline Pipe flow & $\begin{array}{l}\text { Velocity distribution over } \\
\text { cross-section }\end{array}$ & Fully turbulent & $\begin{array}{l}\text { Universal SS applies to } \\
\text { smooth and rough } \\
\text { circular straight pipes }\end{array}$ & Taylor (1954) \\
\hline Planar jet & $\begin{array}{l}\text { Mean velocity and velocity } \\
\text { fluctuation profiles }\end{array}$ & $\begin{array}{l}\Delta y u / v= \\
30,000\end{array}$ & $x / d>40$ & $\begin{array}{l}\text { Gutmark and } \\
\text { Wygnanski } \\
(1976) \\
\end{array}$ \\
\hline $\begin{array}{l}\text { Planar mixing } \\
\text { layer }\end{array}$ & $\begin{array}{l}\text { Mean velocity and velocity } \\
\text { fluctuation profiles }\end{array}$ & $\begin{array}{l}\text { Not available, } \\
\text { nozzle exit } \\
\text { speed } 8 \mathrm{~m} / \mathrm{s}\end{array}$ & $\begin{array}{l}\text { Self-similar profiles are } \\
\text { not universal, as profiles } \\
\text { may depend on the state } \\
\text { of the initial boundary } \\
\text { layer and/or the initial } \\
\text { flow conditions }\end{array}$ & $\begin{array}{l}\text { Champagne et } \\
\text { al. (1976) }\end{array}$ \\
\hline $\begin{array}{l}\text { Planar wake } \\
\text { downstream of a } \\
\text { circular cylinder, } \\
\text { screens and solid } \\
\text { strip, flat plate } \\
\text { and symmetrical } \\
\text { aerofoil }\end{array}$ & $\begin{array}{l}\text { Mean velocity defect } \\
\text { profiles (Fig. 4) }\end{array}$ & $\begin{array}{l}1,360 \leq \\
\Delta y u_{\infty} / v \leq \\
6,500\end{array}$ & $\begin{array}{l}\text { Velocity defects } 0.03- \\
0.15 u_{\infty} ; \text { SS within } \\
\text { individual wake } \\
\text { generators, but no } \\
\text { universality; } \\
\text { measurements at } 100 \leq \theta \\
\leq 2,000\end{array}$ & $\begin{array}{l}\text { Wygnanski et } \\
\text { al. (1986) }\end{array}$ \\
\hline
\end{tabular}




\begin{tabular}{|c|c|c|c|c|}
\hline Plumes & Mean velocity profile & $d u / v \geq 600$ & $\begin{array}{l}\text { For } z / d>50 \text { the } \\
\text { parameter } \alpha_{e} \text { becomes } \\
\text { constant }\end{array}$ & $\begin{array}{l}\text { Carazzo et al. } \\
(2006)\end{array}$ \\
\hline $\begin{array}{l}\text { Rotor wake } \\
\text { vortex }\end{array}$ & $\begin{array}{l}\text { Vorticity and azimuthal } \\
\text { velocity profiles }\end{array}$ & $\begin{array}{l}500 \leq d u /(2 v) \\
\leq 2,000\end{array}$ & Based on DNS & $\begin{array}{l}\text { Ali and Abid } \\
\text { (2014) }\end{array}$ \\
\hline $\begin{array}{l}\text { Shear-driven } \\
\text { entrainment into } \\
\text { a linearly } \\
\text { stratified fluid }\end{array}$ & $\begin{array}{l}\text { Growth of boundary layer } \\
\text { (Fig. 6), mean buoyancy, } \\
\text { mean horizontal velocity, } \\
\text { buoyancy flux, momentum } \\
\text { flux }\end{array}$ & $\begin{array}{l}36 \leq u^{* 2} /(N v) \\
\leq 1,214\end{array}$ & $\begin{array}{l}\text { Proof of entrainment law } \\
e / u^{*} \propto \mathrm{Ri}^{-1 / 2} \text { with } \\
\text { dimensional arguments }\end{array}$ & $\begin{array}{l}\text { Jonker et al. } \\
\text { (2013) }\end{array}$ \\
\hline $\begin{array}{l}\text { Turbulence in } \\
\text { quasi-2D }\end{array}$ & $\begin{array}{l}\text { PDFs for longitudinal } \\
\text { velocity differences }\end{array}$ & Sufficient high & $\begin{array}{l}\text { For inertial subrange } \\
E(k) \sim \kappa^{-5 / 3} \text { (Kolmogorov } \\
-5 / 3 \text { spectrum) }\end{array}$ & $\begin{array}{l}\text { Kolmogorov } \\
\text { (1941), Pope } \\
(2000) \\
\end{array}$ \\
\hline $\begin{array}{l}\text { Turbulence in } \\
\text { quasi-2D rapidly } \\
\text { rotating fluid }\end{array}$ & $\begin{array}{l}\text { PDFs for longitudinal } \\
\text { velocity differences }\end{array}$ & $\lambda_{g} \overline{u^{\prime}} / v=360$ & $\begin{array}{l}\text { For inertial subrange } \\
E(k) \sim \kappa^{-2} \text { (anomaly to } \\
\text { Kolmogorov }-5 / 3 \\
\text { spectrum) }\end{array}$ & $\begin{array}{l}\text { Baroud, Plapp, } \\
\text { She, and } \\
\text { Swinney } \\
(2002)\end{array}$ \\
\hline $\begin{array}{l}\text { Turbulent open } \\
\text { channel air-water } \\
\text { flow on a stepped } \\
\text { chute }\end{array}$ & $\begin{array}{l}\text { Distributions of void } \\
\text { fraction (Fig. 7), bubble } \\
\text { count rate, interfacial } \\
\text { velocity and turbulence } \\
\text { level }\end{array}$ & $\begin{array}{l}380,000 \leq \\
d_{h} u / v \leq \\
710,000\end{array}$ & & $\begin{array}{l}\text { Chanson and } \\
\text { Carosi (2007) }\end{array}$ \\
\hline
\end{tabular}

* For explanations of symbols and abbreviations see Notation; note that studies conducted at a larger $\mathrm{R}$ than investigated are also expected to be self-similar 
Table 3 Phenomena and quantities involving RI with limitations and references

\begin{tabular}{|c|c|c|c|c|}
\hline Phenomenon & $\mathrm{R}$ invariant quantity & $\begin{array}{l}\text { Investigated } \\
R \text { range }\end{array}$ & Comment & Reference \\
\hline $\begin{array}{l}\text { Cross-flow } \\
\text { turbine }\end{array}$ & $\begin{array}{l}\text { Mean power coefficient } \\
\text { (Fig. 11) }\end{array}$ & $\begin{array}{l}d u_{\infty} / v \geq \\
800,000\end{array}$ & $\begin{array}{l}\text { Tests conducted for } \\
300,000 \leq d u_{\infty} / v \leq \\
1,300,000\end{array}$ & $\begin{array}{l}\text { Bachant and } \\
\text { Wosnik } \\
(2016)\end{array}$ \\
\hline Gravity current & $\begin{array}{l}\text { Mixing processes in } \\
\text { current front (Fig. 13) }\end{array}$ & $\begin{array}{l}1,000 \leq \\
h_{5}\left(g^{\prime} q\right)^{1 / 3} / v \leq \\
2,012\end{array}$ & $\begin{array}{l}\text { Applies to most energetic } \\
\text { region; no RI for } \\
h_{5}\left(g^{\prime} q\right)^{1 / 3} / v<1,000\end{array}$ & $\begin{array}{l}\text { Parsons and } \\
\text { García (1998) }\end{array}$ \\
\hline Pipe flow & $\begin{array}{l}\text { Energy head losses } \\
\text { (Moody diagram) }\end{array}$ & $d u / v \geq 50,000$ & $\begin{array}{l}\text { This } \mathrm{R} \text { value applies for } \\
k / d \geq 0.07 \text {, but } \mathrm{R} \text { needs } \\
\text { to be larger for } k / d<0.07\end{array}$ & Massey (1989) \\
\hline $\begin{array}{l}\text { Rough horizontal } \\
\text { cylinder in steady } \\
\text { flow }\end{array}$ & Drag coefficient $C_{D} \approx 1.2$ & $\begin{array}{l}75,000 \leq d u / v \\
\leq 480,000\end{array}$ & $\begin{array}{l}d=0.21 \text { and } 0.5 \mathrm{~m}, \\
\text { effective roughness } k / d= \\
0.038\end{array}$ & $\begin{array}{l}\text { Chaplin and } \\
\text { Subbiah } \\
(1997)\end{array}$ \\
\hline Shields diagram & Critical Shields stress & $d_{g} u^{*} / v>400$ & & Shields (1936) \\
\hline $\begin{array}{l}\text { Wall-bounded } \\
\text { turbulence }\end{array}$ & $\begin{array}{l}\text { Mean flow and Reynolds } \\
\text { shear stresses in 2D } \\
\text { channel flow }\end{array}$ & $\begin{array}{l}1,000 \leq \\
w u^{* /(2 v) \leq} \\
6,000\end{array}$ & & $\begin{array}{l}\text { Schultz and } \\
\text { Flack (2013) }\end{array}$ \\
\hline Wall turbulence & $\begin{array}{l}\text { Wake factor in the law of } \\
\text { the wall/wake }\end{array}$ & $\begin{array}{l}\theta u_{\infty} / v \geq \\
15,000 ; d u / v \geq \\
400,000\end{array}$ & $\begin{array}{l}\text { The first criterion is for } \\
\text { boundary layers and the } \\
\text { second for pipe flow }\end{array}$ & $\begin{array}{l}\text { Smits, } \\
\text { McKeon, and } \\
\text { Marusic } \\
(2011) \\
\end{array}$ \\
\hline $\begin{array}{l}\text { Water treatment } \\
\text { tank (contact tank } \\
\text { under complete } \\
\text { mixing) }\end{array}$ & $\begin{array}{l}\text { Dispersion and mixing } \\
\text { processes (Fig. 12) }\end{array}$ & Not available & $\begin{array}{l}\text { Turbulent flow for } R> \\
4,000\end{array}$ & $\begin{array}{l}\text { Teixeira and } \\
\text { Rauen (2014) }\end{array}$ \\
\hline
\end{tabular}




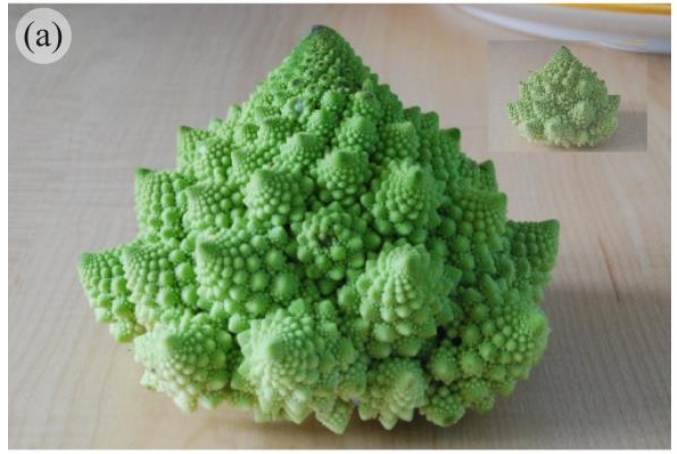

(b)

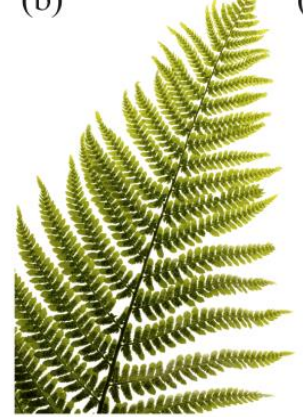

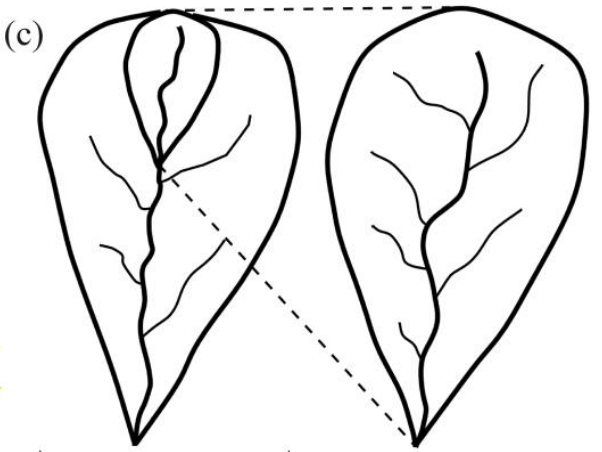

Figure 1 Examples of geometrical SS in nature: (a) Romanesco broccoli

(http://www.butterflyeffect.ca/Close/Pages/SelfSimilarity.html, accessed on 02.08.2016), (b) fern (http://www.ck12.org/geometry/Self-Similarity/lesson/Self-Similarity/, accessed on 02.08.2016) and (c) river networks (Dodds \& Rothman, 2000)

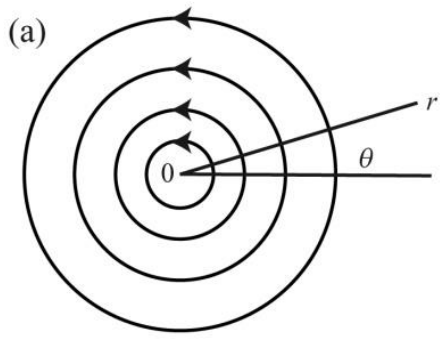

(b)

Figure 2 Vortex: (a) initial condition of idealised irrotational vortex in polar coordinates and (b) real intake vortex at Denison Dam, Lake Texoma (http://www.jutarnji.hr/jezero-iz-zone-sumrakaogroman-vrtlog-nemilice-guta-vodu--vojska-zabranila-pristup-brodovima/1372254/, accessed on 02.08.2016)
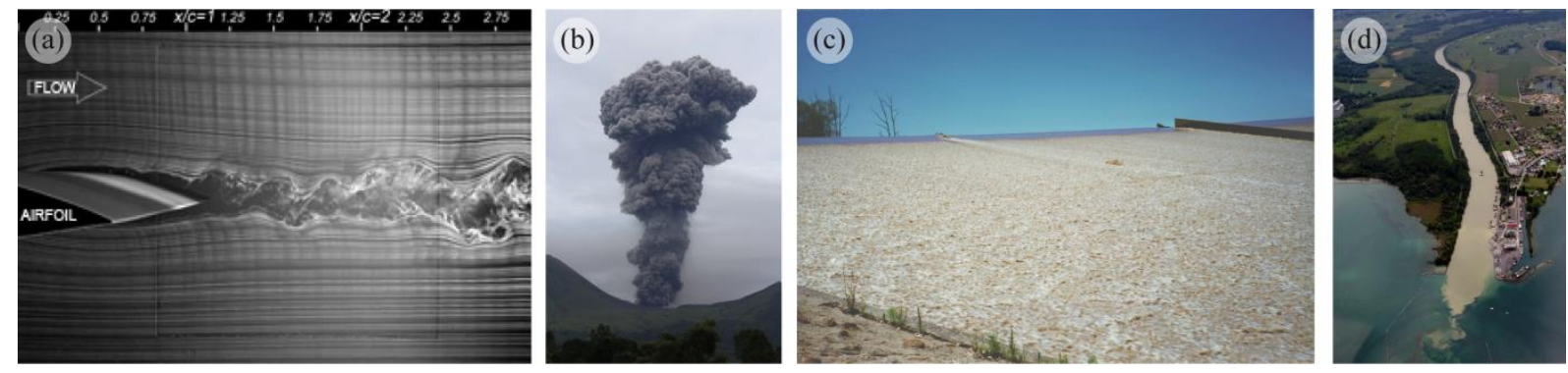

Figure 3 Phenomena involving SS at large R: (a) wake behind an aerofoil

(http://www.fmrl.uwaterloo.ca/research.html, accessed on 02.08.2016), (b) volcanic plume (https://volcanicdegassing.wordpress.com/tag/ash-plume/, accessed on 02.08.2016), (c) turbulent airwater mixture on Chinchilla weir spillway (Australia) in operation on 8 November 1997 at a low flow (Toombes \& Chanson, 2007, with permission of Springer and by courtesy of H. Chanson) and (d) suspended sediment transport down Rhone River discharging into Lake Geneva (http://www.fondriest.com/environmental-measurements/parameters/hydrology/sediment-transportdeposition/, accessed on 02.08.2016) 

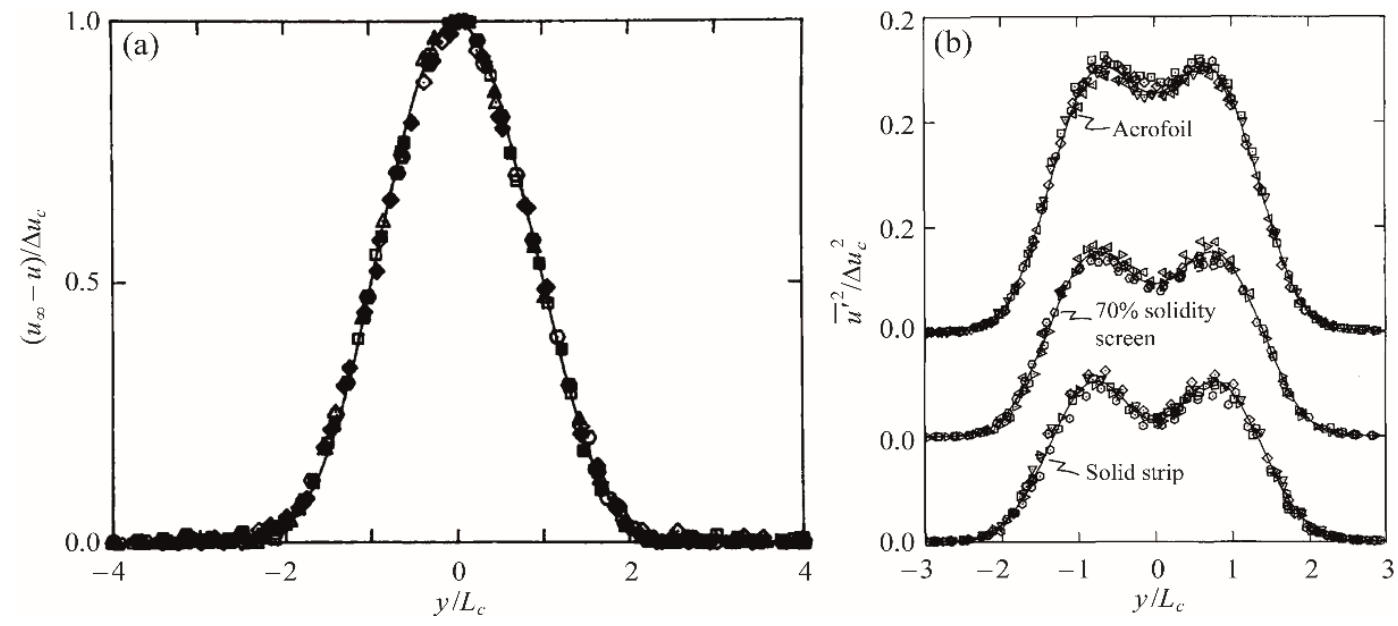

Figure 4 Self-similar features in turbulent wakes: (a) normalised mean velocity defect $\left(u_{\infty}-u\right) / \Delta u_{c}$ versus normalised cross-flow coordinate $y / L_{c}$ downstream of a $30 \%$ solidity screen for $200<(x-$ $\left.x_{0}\right) / 2 \theta<700$ and (b) normalised Reynolds stresses $\bar{u}^{\prime 2} / \Delta u_{c}^{2}$ versus $y / L_{c}$ for a solid strip, $70 \%$ solidity screen and symmetric aerofoil with symbols referring to different downstream locations (Wygnanski et al., 1986)

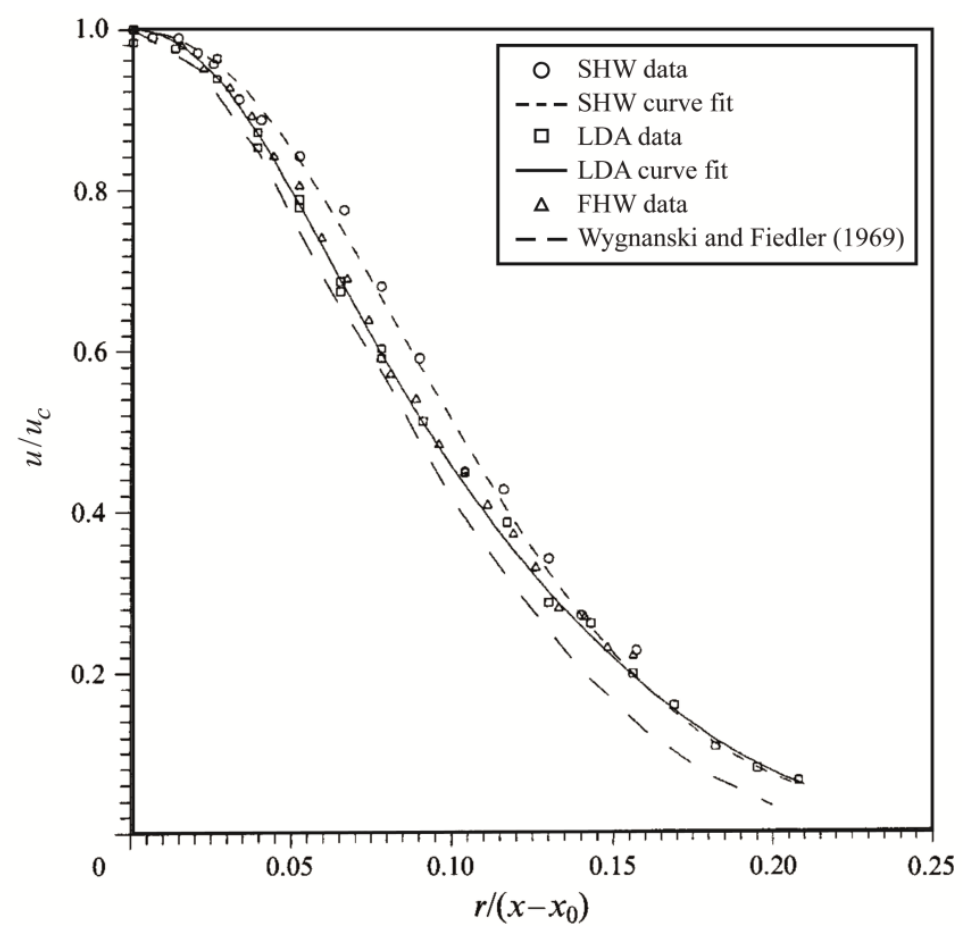

Figure 5 Mean velocity profile of axisymmetric turbulent jet with centreline velocity $u_{c}=B M_{0}{ }^{1 / 2} /(x-$ $x_{0}$ ), a constant $B$ depending on jet exit conditions and momentum flux at jet source $M_{0}$; measurements were conducted with stationary hot-wire (SHW), flying hot-wire (FHW) and laser-Doppler anemometry (LDA) (Hussein et al., 1994) 

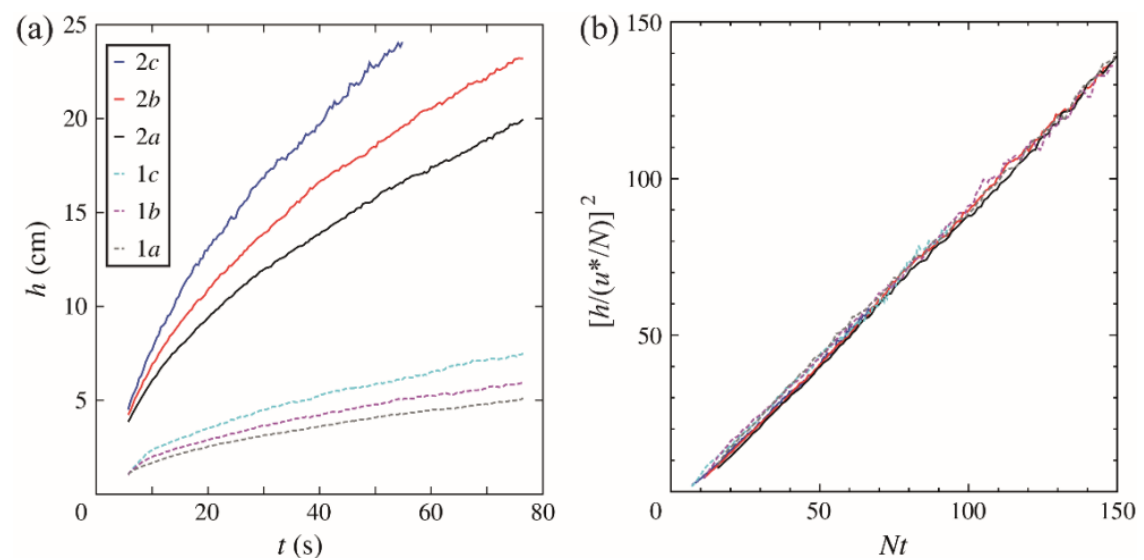

Figure 6 Growth of shear-driven boundary layers into a linearly stratified fluid: (a) mixed layer depth evolution $h(t)$ for six experiments with buoyancy frequencies $N$ and shear velocities $u^{*}$ and (b) collapse of data on a straight line in dimensionless form (Jonker et al., 2013)

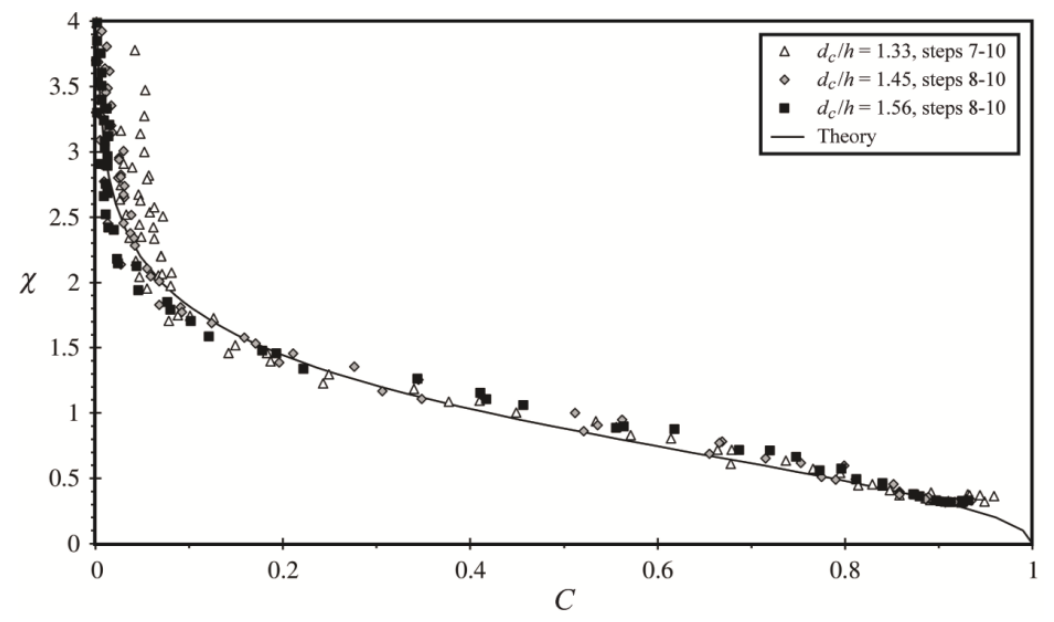

Figure 7 SS in air-water skimming flow on a stepped chute described with analytical solution of advective diffusion equation for air bubbles (Theory): dimensionless void fraction distribution $C(\chi)=$ $1-\tanh ^{2}(\chi)$ with $\left.\chi=K-Z_{90} /\left(2 D_{0}\right)+\left(Z_{90}-1 / 3\right)^{3} / 3 D_{0}\right) ; C=$ void fraction, $K=\tanh ^{-1}\left(0.1^{1 / 2}\right)+1 /\left(2 D_{0}\right)-$ $8 /\left(81 D_{\mathrm{o}}\right), Z_{90}=z / h_{90}=$ dimensionless distance normal to chute, $h_{90}=$ depth where $C=90 \%$ and $D_{\mathrm{o}}=$ dimensionless constant in function of mean $C$ (Chanson \& Carosi, 2007, with permission of Springer)

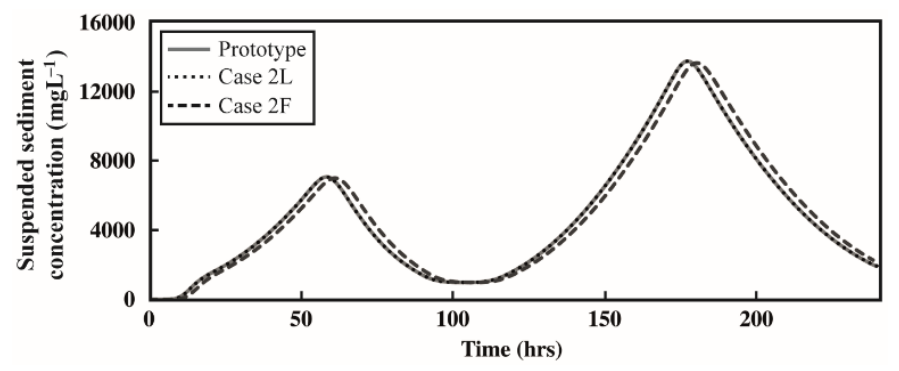

Figure 8 Suspended sediment concentration over time for prototype values (Prototype), for up-scaled test case based on Lie group scaling (Case 2L) and for up-scaled test case based on traditional Froude modelling (Case 2F) (Carr et al., 2015, with permission from ASCE) 


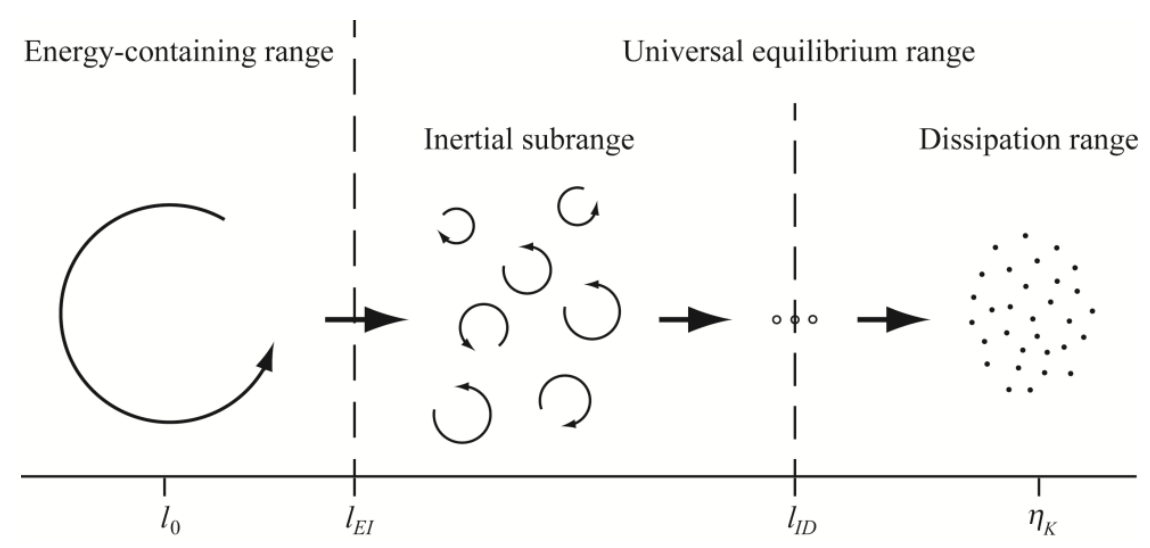

Figure 9 Energy cascade model with both energy-containing and universal equilibrium ranges
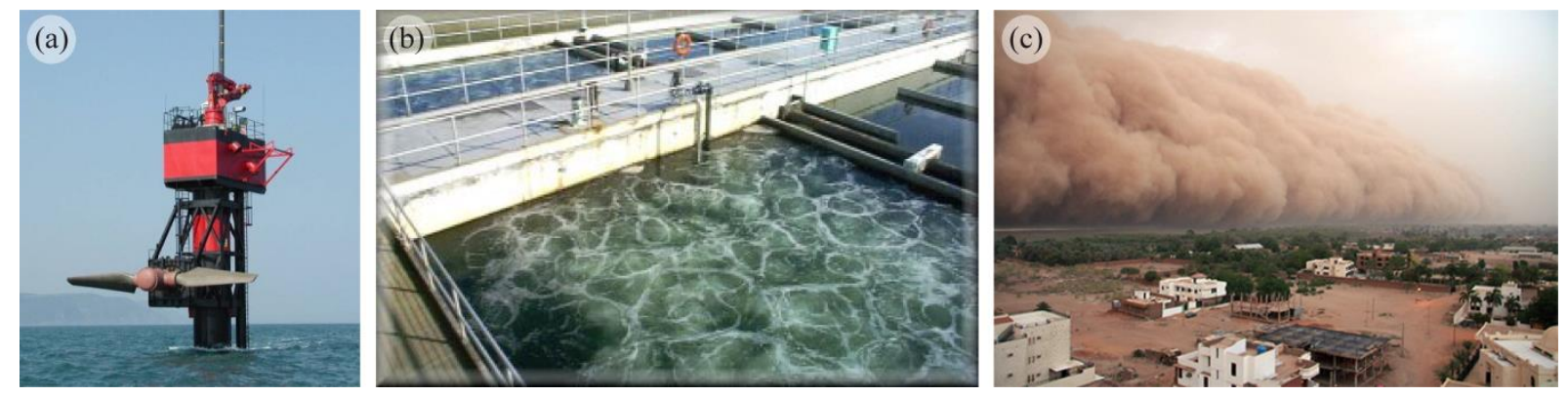

Figure 10 Phenomena involving RI: (a) horizontal axis tidal turbine

(http://tidalturbines.wikispaces.com/Tidal+Current+Turbines, accessed on 02.08.2016), (b) solute transport in a chlorine contact tank (http://www.cortlandwastewater.org/Cl2ContactFrame.html, accessed on 02.08.2016) and (c) gravity current in the atmosphere in Khartoum, Sudan (http://www.techeblog.com/index.php/tech-gadget/5-fascinating-things-about-gravity-currentpowered-haboobs, accessed on 02.08.2016)

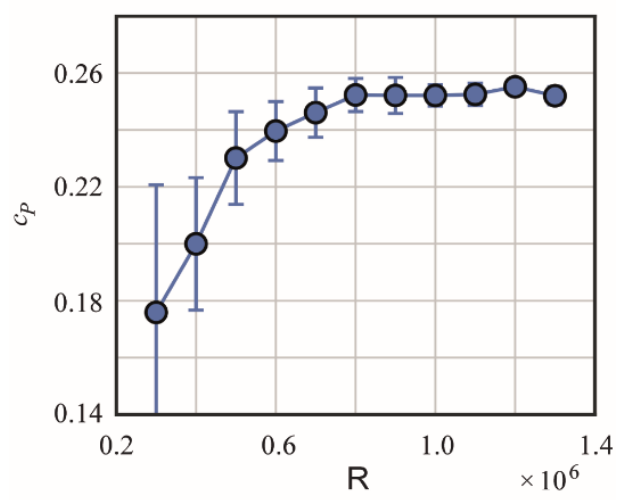

Figure 11 Asymptotically approached $\mathrm{R}$ invariant power coefficient $c_{P}$ level for a TEC (Bachant \& Wosnik, 2016) 

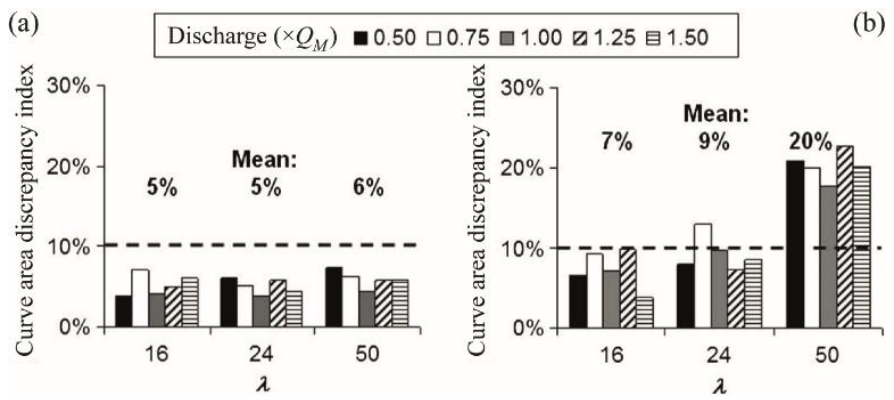

Figure 12 Complete mixing in a contact tank: variation of curve area discrepancy index with scale and discharge for (a) complete mixing and (b) plug flow (Teixeira \& Rauen, 2014, with permission from ASCE)

(a)

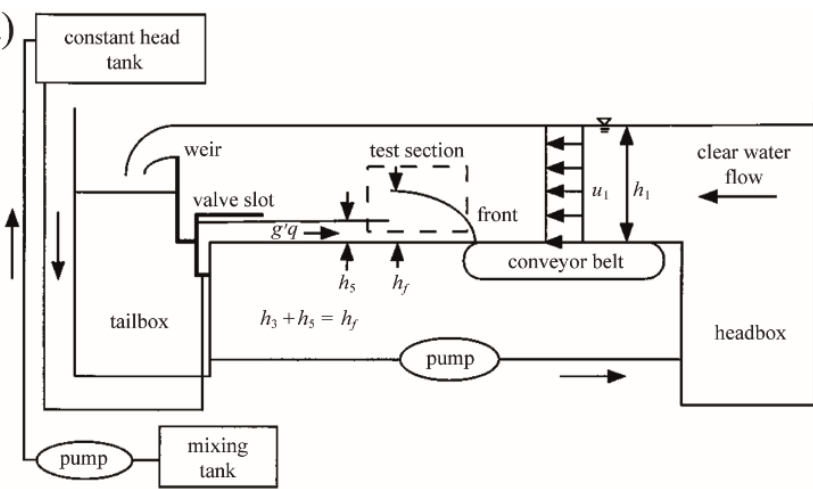

(b)

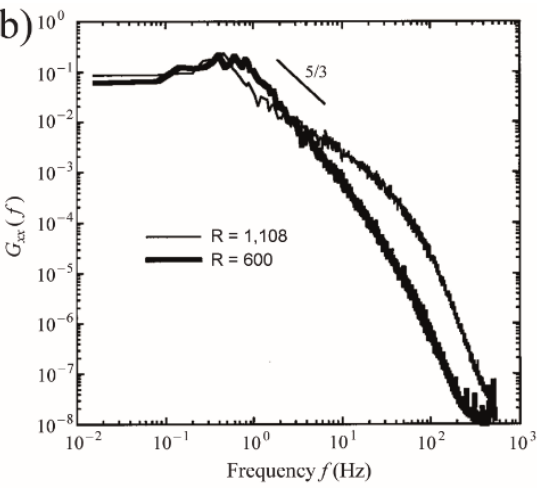

Figure 13 Gravity current investigated with (a) set-up based on arrested gravity current method and (b) power spectra $G_{x x}(f)$ revealing deviations of low from high $\mathrm{R}$ (self-similar) flow data measured in most energetic region at current front (reproduced from Parsons \& García, 1998, with the permission of AIP Publishing) 